\title{
Contribution to the knowledge of the fauna of rove beetles of the subfamily Omaliinae MacLeay, 1825 (Coleoptera: Staphylinidae) of Kunashir Island, Kurile Islands
}

\section{Вкцад в познание фауны жкуков-стафицинид подсемейства Omaliinae MacLeay, 1825 (Coleoptera: Staphylinidae) острова Кунашир, Курильские острова}

\author{
A.V. Shavrin ${ }^{1}$, K.V. Makarov ${ }^{2}$ \\ A.B. Шаврин ${ }^{1}$, K.B. Макаров ${ }^{2}$
}

\footnotetext{
${ }^{1}$ Institute of Life Sciences and Technologies, Daugavpils University, Vienibas 13, Daugavpils LV-5401, Latvia. Email: ashavrin@hotmail.com

${ }^{2}$ Zoology and Ecology Department, Moscow Pedagogical State University, Kibalchicha str. 6, Bld. 5, Moscow 129164, Russia. Email: kvmac@inbox.ru

${ }^{2}$ Московский государственный педагогический университет, кафедра зоологии и экологии, ул. Кибальчича 6, корп. 3, Москва 129164, Россия.
}

KEY WORDS. Staphylinidae, Omaliinae, Kurile Islands, Kunashir Island, fauna, lectotype designation.

КЛЮЧЕВЫЕ СЛОВА. Staphylinidae, Omaliinae, Курильские острова, остров Кунашир, фауна, лектотип.

ABSTRACT. The list of 19 species of 14 genera in four tribes of the rove beetles (Staphylinidae) of the subfamily Omaliinae MacLeay, 1825 (Coleoptera: Staphylinidae) of Kunashir Island is provided. A lectotype is designated for Lesteva plagiata Sharp, 1889. The aedeagi of Lesteva nipponica Watanabe, 1990, L. plagiata Sharp, 1889, Liophilydrodes puncticollis (Nakane et Sawada, 1956), Mannerheimia yasudai Watanabe, 1990, and the aedeagus, mouthparts, and apical segments of the male abdomen of Archaeoboreaphilus miyamorii (Watanabe, 1988) are figured. Habitus photographs of eight species are provided. Additional material of seven species is recorded from adjacent territories. Several first records are reported: Acidota daisetsuzana Watanabe, 1990 (from Kunashir and Maritime Province), Lesteva nipponica, Mannerheimia yasudai, Archaeoboreaphilus miyamorii, Eudectus rufulus Weise, 1877 (all from Kunashir) from Russia, Liophilydrodes puncticollis from Kunashir, Shikotan Islands, and Khabarovsk Territory, Lesteva plagiata, Acrolocha miyamorii, Phloeostiba plana (Paykull, 1792), and Phloeonomus pusillus (Gravenhorst, 1806) from Kunashir, Geodromicus nipponensis (Watanabe, 1990) from Shikotan, and Phloeonomus orientalis Smetana, 1981 from Maritime Province. The features of the fauna of Kunashir Island are briefly discussed. The distributions of all identified species in Kunashir Island are mapped.
РЕЗЮМЕ. Приведён список 19 видов из 14 родов и четырёх триб стафилинид (Staphyinidae) подсемейства Omaliinae MacLeay, 1825 (Coleoptera: Staphylinidae) острова Кунашир. Для Lesteva plagiata Sharp, 1889 выделен лектотип. Приведены рисунки эдеагусов Lesteva nipponica Watanabe, 1990, L. plagiata Sharp, 1889, Liophilydrodes puncticollis (Nakane et Sawada, 1956), Mannerheimia yasudai Watanabe, 1990, а также рисунки эдеагуса, ротовые части и апикальные сегменты брюшка Archaeoboreaphilus miyamorii (Watanabe, 1988). Приведены габитуальные фотографии восьми видов. Для семи видов приведен дополнительный материал из соседних территорий. Несколько видов впервые указаны для разных регионов: Acidota daisetsuzana Watanabe, 1990 (о-в Кунашир и Приморский край), Lesteva nipponica, Mannerheimia yasudai, Archaeoboreaphilus miyamorii, Eudectus rufulus Weise, 1877 (все с Кунашира) для России, Liophilydrodes puncticollis c Кунашира, Шикотана и Хабаровского края, Lesteva plagiata, Acrolocha miyamorii, Phloeostiba plana (Paykull, 1792) и Phloeonomus pusillus (Gravenhorst, 1806) с Кунашира, Geodromicus nipponensis (Watanabe, 1990) с Шикотана и Phloeonomus orientalis Smetana, 1981 из Приморского края. Кратко охарактеризованы особенности фауны Omaliinae о-ва Кунашир. Для всех идентифицированных видов приведены карты по распространению на о-ве Кунашир.

How to cite this article: Shavrin A.V., Makarov K.V. 2019. Contribution to the knowledge of the fauna of rove beetles of the subfamily Omaliinae MacLeay, 1825 (Coleoptera: Staphylinidae) of Kunashir Island, Kurile Islands // Russian Entomol. J. Vol.28. No.1. P.36 -53. doi: 10.15298/rusentj.28.1.06 


\section{Introduction}

With more than 1500 species in 117 genera [Thayer, 2016], the subfamily Omaliinae MacLeay, 1825 is a relatively large group of the rove beetles (Coleoptera: Staphylinidae) distributed in all geographic regions of the world. Little is known about the omaliine fauna of the Russian Far East. Special publications for this region are lacking and numerous taxonomic and faunistic data are scattered in several faunistic studies [Kryzhanovskiy et al., 1973; Molodova, 1973; Shavrin, Gildenkov, 2009; etc.], catalogues [Ryabukhin, 1999; Shibata et al., 2013; etc.], reviews of genera [Ryabukhin, 1991; Shavrin, 2013, 2016; etc.], revisions [Shavrin, 2018; Zerche, 1990, 1993, 2003], etc. To date, about 60 species in 24 genera and 4 tribes are known from this vast territory of Russia [Ryabukhin, 1999; Schülke, Smetana, 2015; etc.]. However, many faunistic records require confirmation and some obscure taxa are in need of taxonomic revision, as a result of which several new species in some genera should be described. There has been no study on the fauna of Omaliinae of Kunashir Island, aside from Naomi et al. [2000], who provide faunistic data for Staphylinidae collected in Kurile Islands (including Kunashir Island) and Kamchatka Peninsula, recording six out of seven species from Kamchatka and Paramushir Island as "Omaliinae sp.”. In the monograph of Krivolutskaja [1973] on the entomofauna of Kurile Islands, 45 species in 25 genera [page 14, Table 1] were reported for this archipelago, without providing a list of species or other details. Filatova \& Lafer [1978] recorded two species from Iturup Island, Geodromicus sp. and Geodromicus beibienkoi Tichomirova, 1973, but both these records require revision. For instance, G. beibienkoi is known only from the mainland of eastern Russia, northwestern China, and North Korea [Shavrin, 2018]. Lafer [1989] recorded Brathinus oculatus Lewis, 1886 from Kunashir Island in his key of the family Brathinidae LeConte, 1861" (transferred to Omaliinae by Hammond [1971]), and later, the same author described Liophilydrodes subtilis iturupensis Lafer, 2004 from Iturup Island [Lafer, 2004]. Zanetti [1993] recorded Eusphalerum prope hidakanum Watanabe, 1990 from Kunashir Island. Shibata et al. [2013] recorded Acrolocha miyamorii Watanabe, 1990 from Kurile Islands. Shavrin [2018] published records of Geodromicus iburinus (Watanabe, 1990), G. lestevoides (Sharp, 1889), and G. nipponensis (Watanabe, 1990) from the same island.

In the present study, a list of 19 species in 14 genera and four tribes of Omaliinae from Kunashir Island is provided. It is based on material collected during field trips by several Russian collectors: S.A. Kurbatov in 1985, 1990 and 2011, A.V. Matalin in 2011, I.V. Melnik in 2011, A.S. Prosvirov in 2009 and 2014, Yu.N. Sundukov in 20132018, by the second author in 1990, 2008, 2011, 2013 and 2017 (see Map 1), and other colleagues. Most of the species are recorded from Kunashir, some also from Russia for the first time. The additional material from adjacent territories yielded new faunistic records of several species. Moreover, a lectotype is designated for Lesteva plagiata
Sharp, 1889, and for some species detailed figures of aedeagi and other body details are provided, which may be useful for species identification. Records of all 14 species identified at the species level are mapped.

Kunashir is the southernmost island of the Greater Kuril Chain. The island is $123 \mathrm{~km}$ long, its width ranging from 7 to $30 \mathrm{~km}$. The relief of the island was formed during acitivities of four volcanoes, which formed three mountain ranges: northern (Tyatya Volcano and Dokuchayev Mts. up to 1819 m a.s.1.), central (Mendeleev Volcano, $888 \mathrm{~m}$ a.s.l.) and southern (Golovnin Volcano, $543 \mathrm{~m}$ a.s.1.). These mountain ranges are divided by the Yuzhnokurilskiy and Sernovodskiy isthmuses (Map 1), both composed of Quaternary marine deposits and volcanogenic, sedimentary and plicate, Neogene rocks. The Kurile Islands are characterized by high rainfall, frequent and excessive fogs, and strong winds. Despite effects of the ocean that significantly affects to seasonally and diurnally fluctuating of the temperature and level of the humidity, combined influences of warm and cold ocean currents, as well as the difficult terrain and different types of the volcanism provides high level of mosaic microclimatic conditions in these islands [Vorobiev, 1963]. The warm Soya Current renders Kunashir the warmest island of the Kuril Archipelago [Razjigaeva et al., 2011]. Owing to the warm climate, high mosaic conditions, and vicinity to Hokkaido, the vegetation of Kunashir Island is considerably richer and more diverse compared to other islands of the arhipelago. More than a thousand species of vascular plants have been recorded [Barkalov, 2009], and extensive spruce-fire, stone birch, and mixed coniferous-broadleaved forests are present. The slopes at 250 to $300 \mathrm{~m}$ a.s.l. are covered with thickets of Siberian dwarf pine (Pinus pumila) and broad-leaf bamboo (Sasa kurilensis). Marshy cereal-sedge meadows and swamps are common for lower reaches of rivers and their estuaries, and floodplains are covered with alder-birch and riparian Salix forests. Despite the small total area of the island and narrow straits separating it from adjacent islands, several endemic and subendemic taxa of beetles are known from Kunashir: the ground beetle Bembidion ruruy Makarov et Sundukov, 2014; the net-winged beetles Plateros krivolutzkii (L.Medvedev, 1966); Punicealis barovskii (L.Medvedev, 1966); the false fire-colored beetle Ischalia kunashirica Nikitsky, 1994; the weevils Shiranoshizo opacus (Zherikhin, 1991) and Lobosoma kurilense Zherikhin, 1991; etc.

The main aim of the present study is an assessment of the omaliine fauna of Kunashir Island and a discussion of the possible ways of its formation.

\section{Material and methods}

When preparing the present paper, we examined more than 200 specimens of Omaliinae, which were collected during about 30 years in several dozens of localities of Kunashir Island (Map 1). All available collecting techniques were applied: hand collecting, aspirators, entomological net sweeping, litter sieving, watering the ground, 
as well as light, pitfall, and window traps. Fourteen species were identified. Some species can not be reliably identified due to the absence of additional material or insufficient knowledge of these taxa at present. Besides that, additional material of several species was examined, which was collected in adjacent territories of the Kuril Archipelago (Shikotan Island), Sakhalin Island, Maritime Province and Khabarovsk Territory of Russia, and in Japan.

The material examined is deposited in:

BMNH - The Natural History Museum, London, UK (R.G. Booth)

CS - collection of Alexey Shavrin, Daugavpils, Latvia IRSNB - Institut Royal des Sciences Naturelles de Belgique, Bruxelles, Belgium (Y. Gérard)

MSPU - Moscow State Pedagogical University, Zoology and Ecology Department, Moscow, Russia (K.V. Makarov)

NHMW - Naturhistorisches Museum Wien, Vienna, Austria (H. Schillhammer)
RCPQ - Russian Centre of Plant Quarantine, Bykovo, Moscow Area, Russia (S.A. Kurbatov)

ZIN - Zoological Institute of the Russian Academy of Sciences, St.-Petersburg, Russia (M.G. Volkovitch) ZMM - Zoological Museum of Moscow University, Moscow, Russia (A.A. Gusakov)

ZMUC - Zoological Museum, University of Copenhagen, København (A.Yu. Solodovnikov)

Morphological studies were carried out using Zeiss Discovery V8 and V12 stereomicroscopes. The habitus photographs were taken with a Canon EOS 5D Mark III camera and a Canon MP-E $65 \mathrm{~mm}$ objective using the extended focus technology. High-resolution habitus images are available at https:/www.zin.ru/Animalia/Coleoptera/rus. All measurements are given in millimeters and were made with a stereoscopic microscope using an ocular micrometer.

In this article we follow the taxonomy provided in Schülke \& Smetana [2015]. In addition to the above

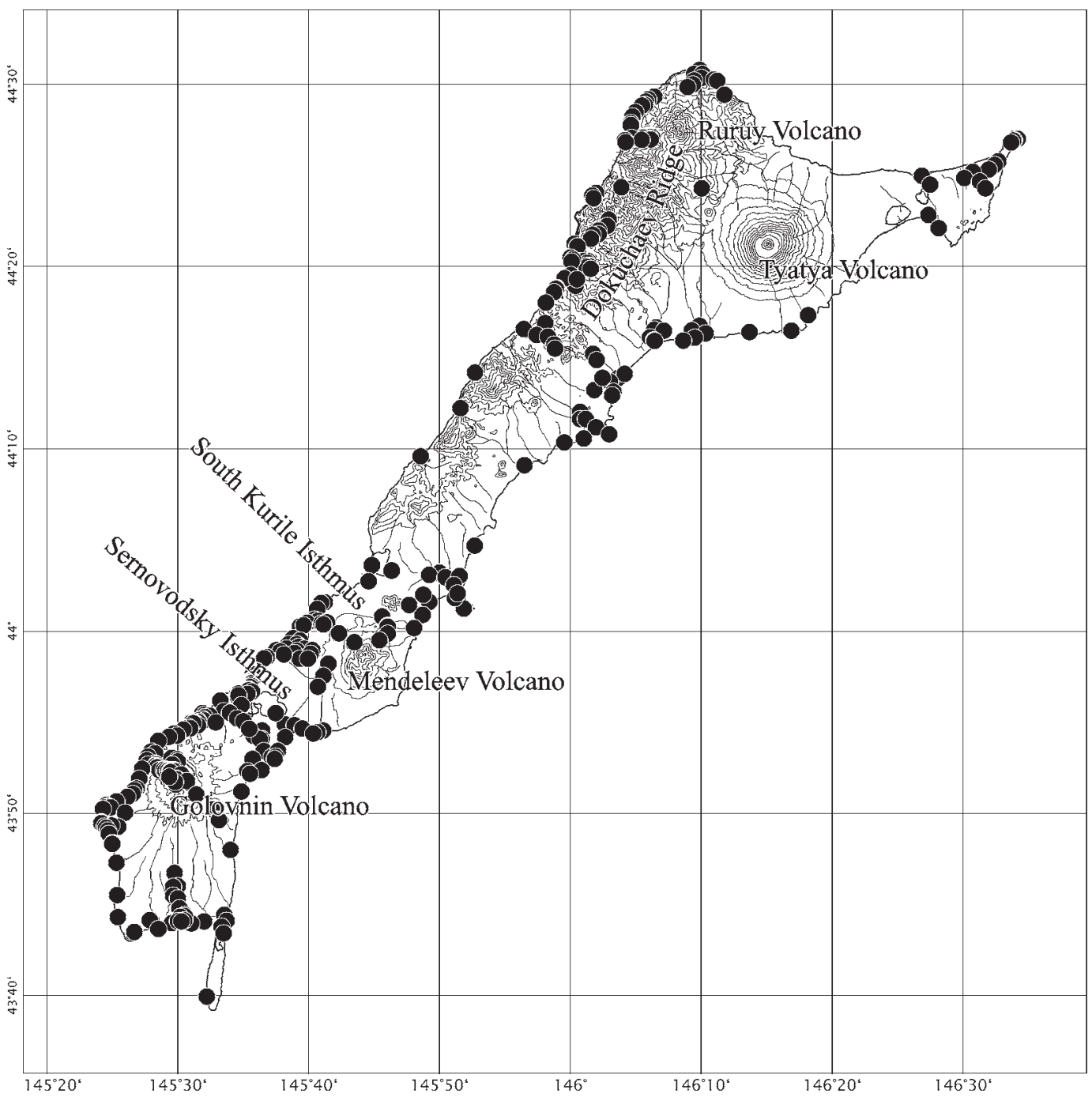

Map 1. Map of localities on Kunashir Island, 2008-2015.

Карта 1. Места сбора на острове Кунашир в 2008-2015 г.г. 
catalogue, distributional data are based on Herman [2001], Shibata et al. [2013], and other catalogues, check-lists, and papers. Relevant taxonomic studies with detailed morphological data, which can be helpful for species identification, are cited for almost every species, and references of faunistic papers are included. Citations of type and historic labels are separated by commas; different lines on labels of type material are
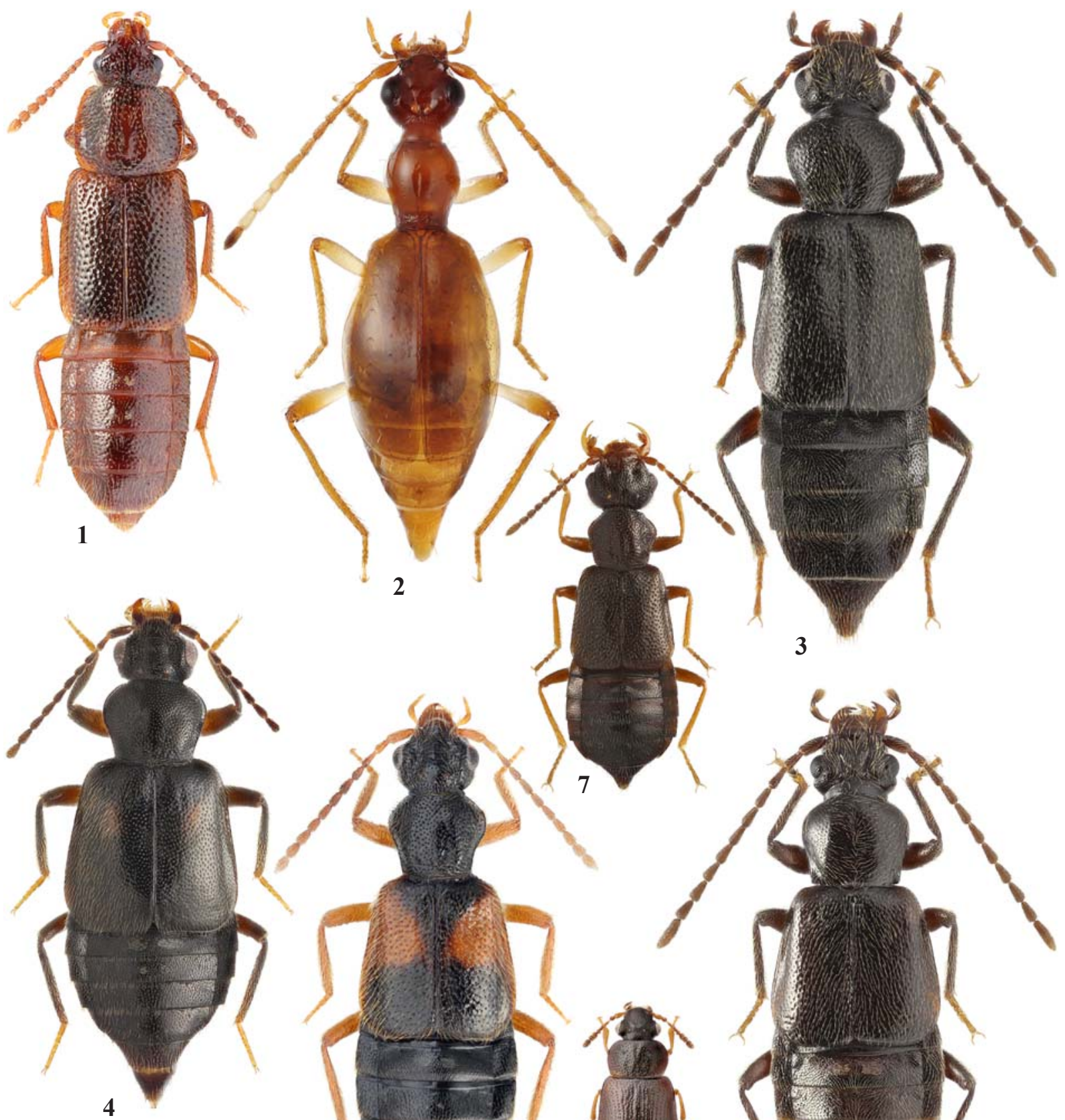

لبللسلبللبللسلبا

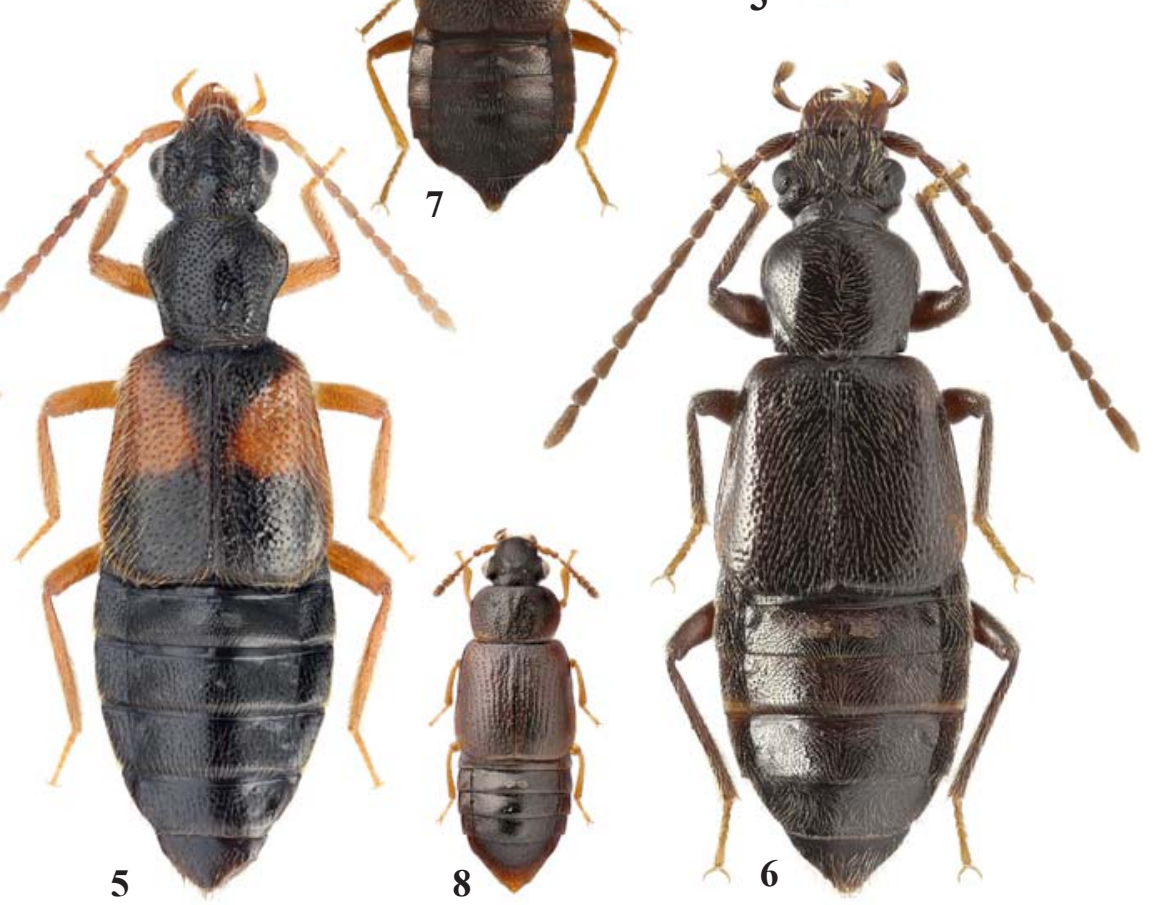

Figs 1-8. Habitus of Omaliinae: 1 - Acidota daisetsuzana Watanabe; 2 - Brathinus oculatus Lewis; 3 - Geodromicus nipponensis (Watanabe); 4 - Lesteva nipponica Watanabe; 5 - L. plagiata Sharp; 6 - Liophilydrodes puncticollis (Nakane et Sawada); 7 Archaeoboreaphilus miyamorii (Watanabe); 8 - Acrolocha miyamorii Watanabe. Scale bar: $1.0 \mathrm{~mm}$.

Рис. 1-8. Внешний вид Omaliinae: 1 - Acidota daisetsuzana Watanabe; 2 - Brathinus oculatus Lewis; 3 - Geodromicus nipponensis (Watanabe); 4 - Lesteva nipponica Watanabe; 5 - L. plagiata Sharp; 6 - Liophilydrodes puncticollis (Nakane et Sawada); 7 Archaeoboreaphilus miyamorii (Watanabe); 8 - Acrolocha miyamorii Watanabe. Масштаб: 1.0 мм. 

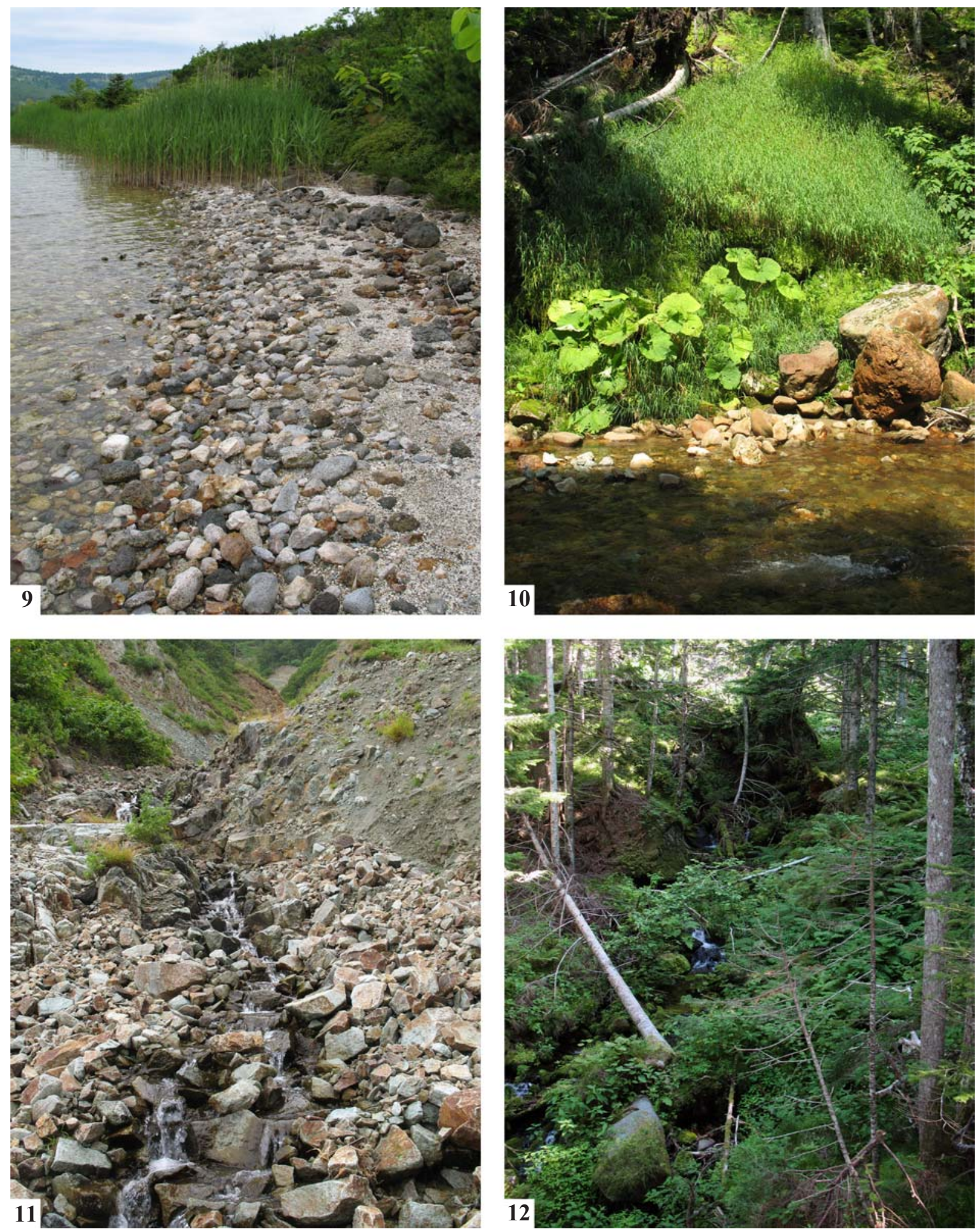

Figs 9-12. Habitats of Omaliinae on Kunashir Island: 9 - shore of Goryachee Lake, calder of Golovnin Volcano (Brathinus oculatus, Liophilydrodes puncticollis); 10 — middle flow of Zolotaya River (Geodromicus iburinus, L. puncticollis); 11 — canyon about $2.5 \mathrm{~km} \mathrm{NW}$ Nelyudimyi Cape (L. puncticollis); 12 - stream ab. 1 km SE Dokuchaevo (Archaeoboreaphilus miyamorii, L. puncticollis). Photo by K.V.Makarov.

Рис. 9-12. Местообитания Omaliinae на острове Кунашир: 9 - берег оз. Горячее, кальдера вулкана Головнина (Brathinus oculatus, Liophilydrodes puncticollis); 10 — среднее течение р. Золотая (Geodromicus iburinus, L. puncticollis); 11 — ущелье 2,5 км С3 мыса Нелюдимый (L. puncticollis); 12 - ручей в 1 км от Докучаево (Archaeoboreaphilus miyamorii, L. puncticollis). Фото К.В. Макарова 
separated by a vertical line. Comments on type labels are given in angled brackets; notes on a label are provided in square brackets.

The distributional maps were created using BmpGen2 [Makarov, 2019]. The coordinates of localities are stored in a database created by Visual FoxPro 9.0.

\section{List of species}

Tribe Anthophagini Thomson, 1859

Acidota daisetsuzana Watanabe, 1990

Fig. 1; Map 2.

Acidota daisetsuzana Watanabe, 1990: 146 (type locality: Japan, Hokkaido, Mt. Kurodake on Daisetsu Mts.)

MATERIAL. $1 \sigma^{7}$ : $19 \mathrm{~km}$ SW Yuzhno-Kurilsk, slope near Mendeleev volcano, 43⒌ $59^{\prime} 53^{\prime \prime} \mathrm{N} 145^{\circ} 42^{\prime} 18^{\prime \prime} \mathrm{E}, 23.09 .2009$, A.S. Prosvirov leg. (CS).

ADDITIONAL MATERIAL. RUSSIA: 1 : Maritime Province, Tetyukhe (Dal'negorsk), 15.07.1973, [without collector name] (ZMM).
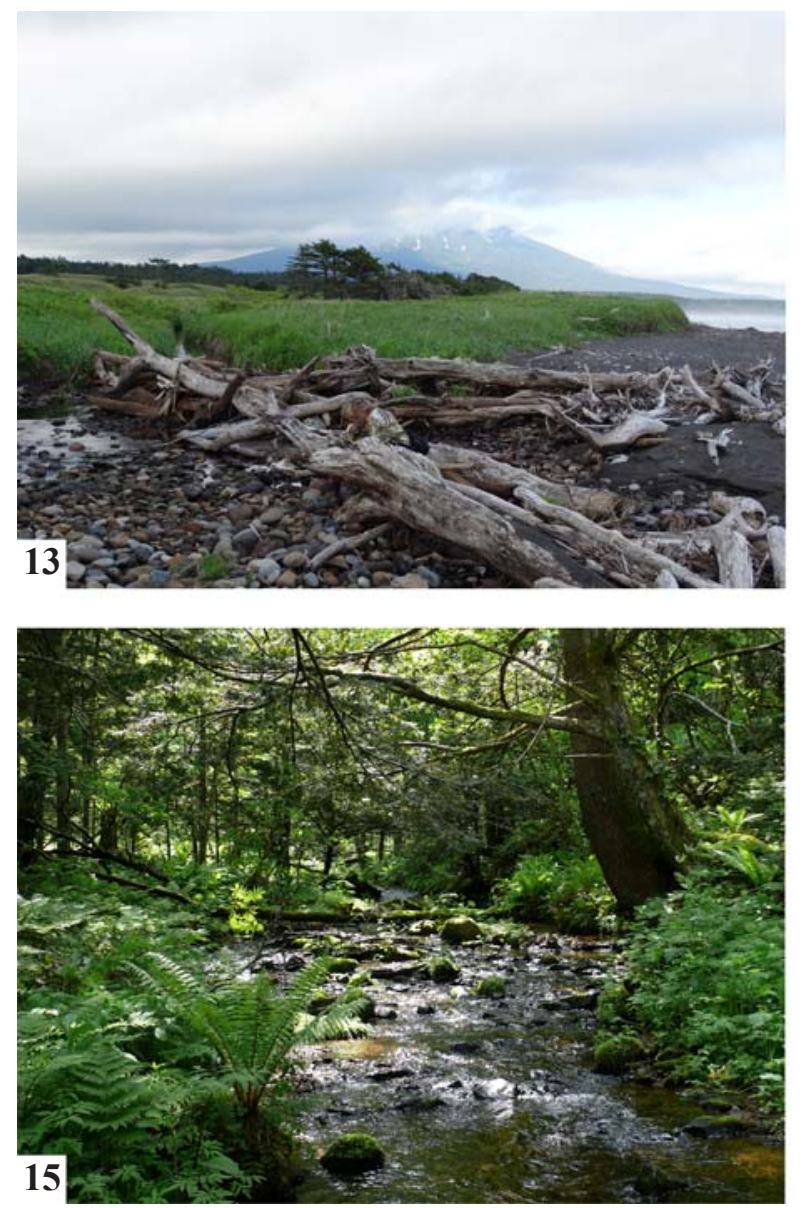

DISTRIBUTION. Russia: Maritime Province, southern Kuriles (Kunashir: Map 2). - Japan (Hokkaido).

BIONOMICS. Detailed ecological data are not available.

REMARKS. Habitus as in Fig. 1. The species is recorded for Kunashir Island and Russia, including mainland territory (Maritime Province), for the first time.

\section{Brathinus oculatus Lewis, 1886}

Figs 2, 9, 13; Map 3 .

Brathinus oculatus Lewis, 1886: 85 (type locality: Japan, Hokkaido, Yezo, district of the Ishikari River); Hammond, 1971: 68; Watanabe, Sato, 1981: 618; Lafer, 1989: 346; Watanabe, 1990: 247; Herman, 2001: 272; Brathinus japonicus Nakane, 1961: 3 (type locality: Japan, Hokkaido, Sapporo); Nakane, 1963: 82.

MATERIAL. 1 specimen: $1.5 \mathrm{~km}$ upper mouth of Severyanka river, $44^{\circ} 19^{\prime} 58^{\prime \prime N} 146^{\circ} 1^{\prime} 15^{\prime \prime} \mathrm{E}, 10.08 .2017$, Yu.N. Sundukov leg. (MSPU); 4 specimens: Saratovskaya River near Tyatina River, $44^{\circ} 16^{\prime} 28^{\prime \prime} N 146^{\circ} 07^{\prime} 09^{\prime \prime}$ E, 5-8.07.2014, Yu.N. Sundukov, L.A. Sundukova leg. (MSPU); 24 specimens: between Saratovskaya and Tyatina Rivers, $44^{\circ} 15^{\prime} 56^{\prime \prime} \mathrm{N} 146^{\circ} 08^{\prime} 37^{\prime \prime}$ E, 21-25.07.2014, Yu.N. Sundukov, L.A. Sundukova leg. (MSPU); 2 specimens: lower reaches of Saratovskaya river, $44^{\circ} 15^{\prime} 57^{\prime \prime N} 148^{\circ} 06^{\prime} 23^{\prime \prime} \mathrm{E}, 2-4.07 .2014$, Yu.N. Sundukov, L.A. Sundukova leg. (MSPU); 1 specimen: same data, 1016.08.2014 Yu.N. Sundukov, L.A. Sundukova leg. (MSPU); 6 speci-
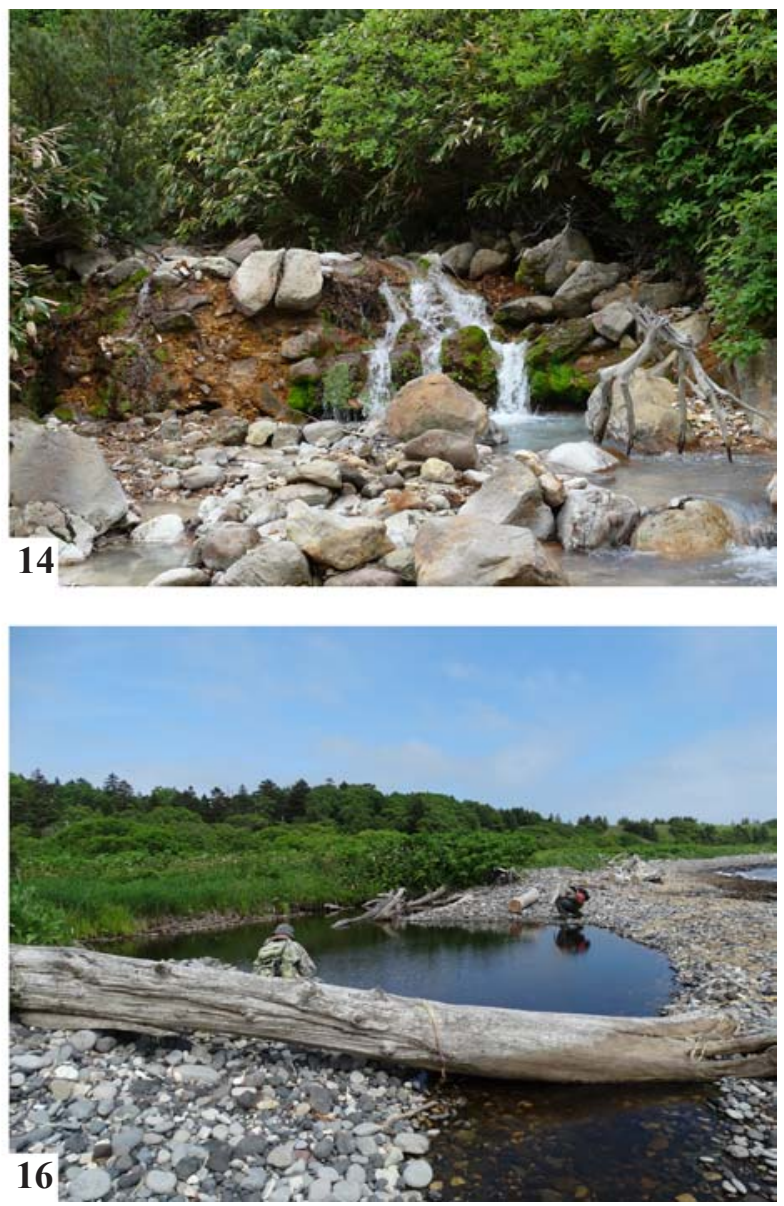

Figs 13-16. Habitats of Omaliinae on Kunashir Island: 13 - shore near Saratovskaya and Tyatina Rivers Sundukov (Brathinus oculatus, Geodromicus nipponensis, Lesteva plagiata); 14 — valley of Kislaya River (Geodromicus iburinus); 15 — environs of Tretyakovo, valley of Valentina Stream (L. plagiata); 16 - Saratovskaya River-Tyatina River, shore near Ploskogornyi Cape (Mannerheimia yasudai); 13, 16 photo by Yu.N. Sundukov; 14, 15 — by A.V. Matalin.

Рис. 13-16. Местообитания Omalinae на острове Кунашир: 13 - побережье близ рек Саратовская и Тятина (Brathinus oculatus, Geodromicus nipponensis, Lesteva plagiata); 14 — долина р. Кислая (Geodromicus iburinus); 15 — долина ручья Валентины, окр. Третьяково (L. plagiata); 16 - берег между реками Саратовская и Тятина близ мыса Плоскогорный (Mannerheimia yasudai); 13, 16 фото Ю.Н. Сундкова; 14, 15 - фото А.В. Маталина. 
mens: lower reaches of Filatova River, 22-27.06.2013, Yu.N.Sundukov leg.; 2 specimens: Aliger Lake, floodland forest of Alnus and Lysichiton, 31.07.1994, Yu.Eskov leg. (MSPU); 6 specimens: cordon Alekhinsky, 28-30.09.2014, Yu.N.Sundukov leg.; 2 specimens: lower reaches of Sernovodka river, swamp, 4354'54"N 145'38'11"E, 10.08.2014, Yu.N Sundukov, L.A. Sundukova leg. (MSPU); 2 specimens: east shore of Glukhoe Lake, 435' $11^{\prime \prime} \mathrm{N} 145^{\circ} 38^{\prime} 12^{\prime \prime}$, 9.07.2008, K.V. Makarov leg (MSPU); 1 O: Alyokhino, 01.06.2011, I.V. Melnik leg. (MSPU); 2 specimens: Alyokhino, 11-14.09.2014, Yu.N. Sundukov leg. (MSPU) 16 specimens: Ozyorniy kordon, 7-9.07.2013, Yu.N. Sundukov, L.A Sundukova (MSPU); $6 \sigma^{\top} \sigma^{\top}, 7$ 우: Ozyorniy kordon, 23-24.04.2018, Yu.N. Sundukov (CS, MSPU); 9 specimens: west shore of Goryacheye Lake, 7-10.07.2017, Yu.N. Sundukov, L.A. Sundukova leg. (MSPU); 3 specimens: southwest shore of Goryacheye Lake, 30.VI-3.07.2016, Yu.N. Sundukov, L.A. Sundukova leg. (MSPU); 2 specimens: south shore of Goryacheye Lake, $43^{\circ} 52^{\prime} 26^{\prime \prime} \mathrm{N} 145^{\circ} 628^{\prime} 58^{\prime \prime} \mathrm{E}-$ $43^{\circ} 52^{\prime} 08^{\prime \prime} \mathrm{N} 145^{\circ} 31^{\prime} 20^{\prime \prime}$ E, 27-29.06.2013, Yu.N. Sundukov, L.A. Sundukova leg. (MSPU); 2 specimens: lower reaches of Ozyornaya river, $43^{\circ} 52^{\prime} 37^{\prime \prime N} 145^{\circ} 28^{\prime} 19^{\prime \prime} \mathrm{E}, 6.09 .2014$, Yu.N. Sundukov leg. (MSPU); 3

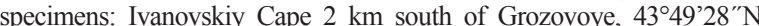
$145^{\circ} 24^{\prime} 09^{\prime \prime} E, 2.06 .2015$, Yu.N. Sundukov leg. (MSPU); 9 specimens: env. Dubovoye, 25-26.07.2017, Yu.N. Sundukov, L.A. Sundukova leg. (MSPU); $3 \sigma^{7} \sigma^{7}, 2$ + 2 : watershed of Golovnin and Khlebinov rivers, 25 29.09.2015, Yu.N. Sundukov leg. (CS, MSPU); 1 \%: [without exact locality] 21.07.1981. D. Emelyanov leg. (CS).

ADDITIONAL MATERIAL. RUSSIA: 1 9: Sakhalin, YuznoSakhalinsk, botanical garden, 16.06.2018, Yu.N. Sundukov leg. (CS); 1 specimen: Yu. Sakhalin, left source of Elanka River, $46^{\circ} 56^{\prime} 50^{\prime \prime} \mathrm{N} 142^{\circ} 48^{\prime} 43^{\prime \prime} \mathrm{E}, 31.07 .2017$, K.V. Makarov leg. (MSPU); 1 specimen: $30 \mathrm{~km}$ W Aniva, Bryanka River, 14.07.1993, A. Pütz, D. Wrase leg. (NHMW); 1 specimen: Aniva district, Kamyshoviy Mts. [without collector name] (NHMW); 1 specimen: Yu. Sakhalin, $14 \mathrm{~km}$ WNW Lesnoe, valley Peskovka river, 46 57'33"N $142^{\circ} 54^{\prime} 34^{\prime \prime}$, 23-30.VI.2003, I. Melnik leg (MSPU).

DISTRIBUTION. Russia: Sakhalin, southern Kurile Islands (Kunashir: Map 3). — Japan (Hokkaido).

BIONOMICS. Brathinus oculatus was collected along banks of clear watercourses with moss-covered stones (Figs $9,13)$. Numerous specimens were collected in debris at the bank of the thermal Goryachee Lake (Fig. 9).

REMARKS. Habitus as in Fig. 2.

\section{Geodromicus iburinus (Watanabe, 1990)}

Figs 10, 14; Map 4.

Psephidonus iburinus Watanabe, 1990: 278 (type locality: Japan, Hokkaido, Kita-Yuzawa, Iburi). Other refernces see in Shavrin [2018].

DISTRIBUTION. Russia: southern Kurile Islands (Kunashir: Map 4). — Japan (Hokkaido).

BIONOMICS. Specimens were collected at the banks of small streams and tributaries of rivers (Figs 10, 14), mainly in the southern part of Kunashir Island, usually near thermal fields of Mendeleev and Golovnin volcanoes.

\section{Geodromicus lestevoides (Sharp, 1889) Map 5.}

Anthophagus lestevoides Sharp, 1889: 470 (type locality: Japan, Hokkaido, Hakodate); Geodromicus lestevoides: Zerche, 2003 280; Shavrin, 2018: 171. Other references see in Shavrin [2018].

DISTRIBUTION. Russia: Amur Area, Khabarovsk Territory, Maritime Province, Sakhalin Area including Kurile Islands (Kunashir: Map 5). — China (Liaoning), Korea, Japan (Hokkaido, Honshu, Shikoku).

BIONOMICS. Detailed ecological data for the specimen collected in Kunashir Island are not available [Shavrin, 2018].

Geodromicus nipponensis (Watanabe, 1990) Figs 3, 13; Map 6.

Psephidonus nipponensis Watanabe, 1990: 275 (type locality: Japan, Honshu: Gunma Prefecture, near Nidoage, Asama Plateau). Other references see in Shavrin [2018].
MATERIAL. $1 \sigma^{7}, 1$ : $1-1.5 \mathrm{~km}$ upper mouth of lower reaches of Severyanka River, 4419'58"N 146 ${ }^{\circ} 1^{\prime} 15^{\prime \prime} \mathrm{E}, 16.06 .2017$, Yu.N. Sundukov leg. (CS); 7 specimens: $2-3 \mathrm{~km}$ of road Rudnoye-Filatovskiy, 16.06.2014, Yu.N. Sundukov leg. (CS, MSPU); 2 specimens: lower reaches of Filatova River, 25-27.06.2013, Yu.N. Sundukov leg. (CS); $1 \sigma^{7}, 4$ कo : valley of Tyatina River, 1-2 km upstream of mouth, 07-10.06.2016, Yu.N. Sundukov leg. (CS, MSPU); 1 specimen: Saratovskaya River near Tyatina River, 21-24.08.2014, Yu.N. Sundukov leg. (CS); 10 specimens: environs of Saratovskiy kordon, 2-4.07.2014, Yu.N. Sundukov leg. (CS, MSPU); $1 \sigma^{7}, 3$ 우: lower reaches of Saratovskaya River, $44^{\circ} 15^{\prime} 46^{\prime \prime} \mathrm{N} 146^{\circ} 06^{\prime} 13^{\prime \prime} \mathrm{E}$, 14-15.07.2014, Yu.N. Sundukov, L.A. Sundukova leg. (CS, MSPU); $1 \sigma^{7}, 1 \sigma^{7}$ : lower reaches of Sernovodka River, $43^{\circ} 54^{\prime} 54^{\prime \prime} \mathrm{N}$ $145^{\circ} 38^{\prime} 11^{\prime \prime}$, swamp, 10.08.2014. Yu.N. Sundukov, L.A. Sundukova leg. (CS).

ADDITIONAL MATERIAL. RUSSIA: $1 \sigma^{\top}, 2$ 우: Sakhalin, Yuzhno-Sakhalinsk, botanical garden, 16.06.2018, Yu.N. Sunduk-

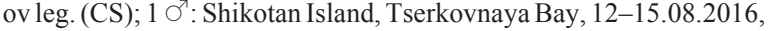
Yu.N. Sundukov leg. (CS).

DISTRIBUTION. Russia: Sakhalin, Kurile Islands (Urup, Kunashir: Map 6, Shikotan). — Japan (Honshu).

BIONOMICS. Specimens were collected on gravel banks of relatively big rivers flowing from Dokuchayev mountain range (Fig. 13); not recorded from thermal zones.

REMARKS. Habitus as in Fig. 3. In the list of the studied material of $G$. nipponensis from Kunashir Island provided by Shavrin [2018] the same four labels were erroneously repeated (page 176), which in fact belong to G. iburinus (page 170). Here, we list material from Kunashir that was used, but not cited by Shavrin [2018]. The species is recorded from Shikotan Island for the first time.

\section{Lesteva nipponica Watanabe, 1990}

Figs 4, 17-18; Map 7.

Lesteva nipponica Watanabe, 1990: 164 (type locality: Japan, Honshu, Nara Prefecture, Mt. Kasuga-yama)

MATERIAL. 1 T': Stolbovskoy Stream above springs, 21.07.2013, K.V. Makarov leg. (CS).

DISTRIBUTION. Russia: southern Kurile Islands (Kunashir: Map 7). — Japan (Honshu).

BIONOMICS. The specimen was collected on a steep slope on the bank of a cold stream near thermal springs.

REMARKS. Habitus as in Fig. 4. In the present study we provide more detailed figures of the aedeagus (Figs 17-18), since Figs 195-197 in Watanabe [1990] are somewhat sketchy. This species is recorded from Russia and Kunashir for the first time.

\section{Lesteva plagiata Sharp, 1889}

Figs 5, 13, 15, 19-20; Map 8.

Lesteva plagiata Sharp, 1889: 472 (type locality: Japan, Honshu, Yokohama, Oyayama, Miyanoshita); Shavrin, Gildenkov, 2009: 122. Other references see in Watanabe [1990] and Herman [2001].

TYPE MATERIAL. Lectotype (here designated) of Lesteva plagiata Sharp, 1889 O': 'Lesteva plag- | iata. Type | D.S. Japan.Lewis' $<$ handwritten on card with beetle, in black India ink $>$, 'Type' < round card with red frame, printed $>$, 'Japan. [underlined by yellow] | G. Lewis.' < rectangular label, printed>, 'Sharp Coll. | 1905-313.' <rectangular label, printed $>$, 'LECTOTYPE | Lesteva | plagiata Sharp, 1889 | Shavrin A.V. des. 2019’< <red rectangular label, printed $>(\mathrm{BMNH})$. Paralectotypes: $2 \sigma^{7} \sigma^{\top}$ [Specimens glued on the same square card]: 'Lesteva plag- | iata. D.S. | Japan.Lewis' $<$ handwritten on card with beetles, in black India ink $>$, 'Japan. [underlined by yellow] | G. Lewis.' < rectangular label, printed>, 'Sharp Coll. | 1905-313.' <rectangular label, printed > BMNH); 2 우: 'Japan. [underlined by yellow] | G. Lewis.' < rectangular label, printed $>$, 'Sharp Coll. | 1905-313.' <rectangular label, printed> (BMNH); 1 O: 'Japan. [underlined by yellow] $\mid$ G. Lewis.' $<$ rectangular label, printed $>$, 'Miyanoshita. | 24.IV.-3.V.80.'<rectangular label printed $>(\mathrm{BMNH}) ; 1$; : 'Japan. [underlined by yellow] $\mid \mathrm{G}$. 
Lewis. | 1910-320.' < rectangular label, printed>, 'Nagasaki. | 22.V.3.VI.[18]81'<rectangular label, printed> $(\mathrm{BMNH}) ; 1$ O': 'Japan. [underlined by yellow] | G. Lewis. | 1910-320. <printed>, 'Yokohama. | 20.III.-14.IV.80.' <rectangular label, printed>, 'Lesteva | plagiata' $<$ rectangular label, printed $>$ (BMNH)

MATERIAL. $10^{7}$ : Saratovskaya River near Tyatina River, $21-$ 24.08.2014, Yu.N. Sundukov leg. (CS); $3 \sigma^{7} \sigma^{7}, 6$ 오: environs of Saratovskiy kordon, 2-4.07.2014, Yu.N. Sundukov leg. (CS, MSPU); $1 \mathrm{O}^{7}$ : environs of Saratovskiy kordon, lower flow of Saratovskaya

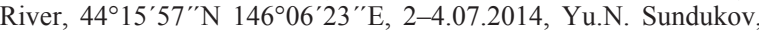
L.A. Sundukova leg. (MSPU); 1 : environs of Tretyakovo, valley of Valentina Stream, 24.05.2011, A.V. Matalin leg. (MSPU); $10^{7}, 7$ 우: Tretyakovo, in litter near stream, 18-21.07.1990, S.A. Kurbatov leg. (CS, RCPQ); 1 ㅇ: Mendeleevo, 04.07.1991, S.A. Kurbatov leg. (ZMM).

ADDITIONAL MATERIAL. RUSSIA: $1 \sigma^{7}, 3$ 우: Maritime Province: 'Seitengraben des Perwaja Rietschka', 'Tales, nördl Wladiwostok', 'H. Frieb leg. 1918-1920', 'plagiata Shp', 'ex coll. Scheerpeltze' (NHMW); JAPAN: 1 +: 'Mt. Daisien Prov. HOKI by M. Suzuki 12-8-1917.' (ZIN); 2 O$^{7} \sigma^{7}$ : 'Kioto Japon' (IRSNB); 2 O $^{7} 0^{7}$,

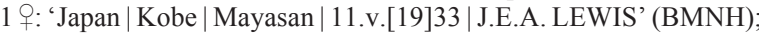
$10^{7}$ : 'JAPAN Kobe', 'Mayasan 27.iv.[19]30', 'Lesteva | plagiata | Shp', 'M.Cameron. Bequest B.M. 1955-147.' (BMNH).

DISTRIBUTION. Russia: Maritime Province, southern Kurile Islands (Kunashir: Map 8). — Japan (Honshu, Shikoku, Kyushu). The record of L. plagiata for South Korea by Cho et al. [2002] is a misidentification of L. miyabi Watanabe, 1990 [Kim, Ahn, 2011].

BIONOMICS. Specimens were collected on stone banks of streams and rivers (Figs 13, 15).

REMARKS. In the original description [Sharp, 1889], the number of syntypes was not specified. The first author designates a lectotype to fix the identity of the name. Lesteva plagiata is a relatively variable species both in habitus and in the shape of the apical portions of the aedeagus and parameres. It is similar to L. kojohamana Watanabe, 1990, described from Iburi, Hokkaido, and a potential synonym of $L$. plagiata. A revision of type material would be required to confirm this. Habitus as in Fig. 5. The aedeagus of L. plagiata in two projections is illustrated in Figs 19-20. The species is recorded from Kunashir Island for the first time.

\section{Liophilydrodes puncticollis (Nakane et Sawada, 1956)} Figs 6, 9-12, 21-26; Map 9.

Philydrodes (Lioplax) puncticollis Nakane et Sawada, 1956: 53 (type locality: Japan, Hokkaido, Nukabira); Shibata, 1965: 317, 1976: 122; Shibata et al., 2013: 75; Phylidrodes [sic!] (Lioplax) puncticollis: Watanabe, Shibata, 1965: 317; Philydrodes puncticollis: Watanabe, 1985: 278; Naomi, 1989: 261; Liophilydrodes puncticollis: Watanabe, 1990: 306; Zerche, 2003: 289

MATERIAL. 3 O' $\sigma^{7}, 2$ 우: about $2.5 \mathrm{~km}$ NW Nelyudimy Cape, 04.08.2013, K.V. Makarov, Yu.N. Sundukov leg. (CS, MSPU); 1 $\sigma^{7}, 1 \sigma^{7}$ : brook, about $1 \mathrm{~km}$ SE Dokuchayevo, 01.08.2013, K.V. Makarov leg. (CS); $1 O^{\prime}$ : Dalniy Stream, 09.08.2013, Yu.N. Sundukov leg. (CS); 1 q: Dokuchayev Ridge, stream N from Vodopad-

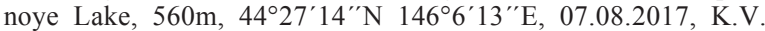
Makarov, Yu.N. Sundukov leg. (MSPU); $1 \sigma^{7}, 1$ \%: same Ridge, stream S from Vodopadnoye Lake, 560, 44 $27^{\prime} 1^{\prime \prime} \mathrm{N} 146^{\circ} 6^{\prime} 4^{\prime \prime} \mathrm{E}$, 06.08.2017, Yu.N. Sundukov leg. (CS, MSPU); $2 O^{7} O^{7}$ : same ridge, stream W from Vodopadnoye Lake, 560m, 44⒉ $7^{\prime} 7^{\prime \prime} \mathrm{N} 146^{\circ} 6^{\prime} 14^{\prime \prime} \mathrm{E}$, 04.08.2017, K.V. Makarov, Yu.N. Sundukov leg. (CS); 2 우: same Ridge, environs of Vodopadnoye Lake, 500m, 44 ${ }^{\circ} 27^{\prime} 6^{\prime \prime} \mathrm{N} 146^{\circ} 6^{\prime} 5^{\prime \prime} \mathrm{E}$, 04-07.08.2017, K.V. Makarov leg. (MSPU); 2 우옹 same Ridge, 1.5 $\mathrm{km}$ N Glukhoy Stream, 0-300, 44 $27^{\prime} 04^{\prime \prime} \mathrm{N} 146^{\circ} 4^{\prime} 40^{\prime \prime} \mathrm{E}, 03.08 .2017$, Yu.N. Sundukov leg. (CS); $1 \mathrm{O}^{7}$ : same Ridge, E slope, Williams

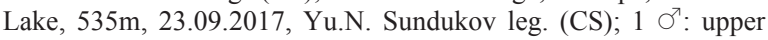
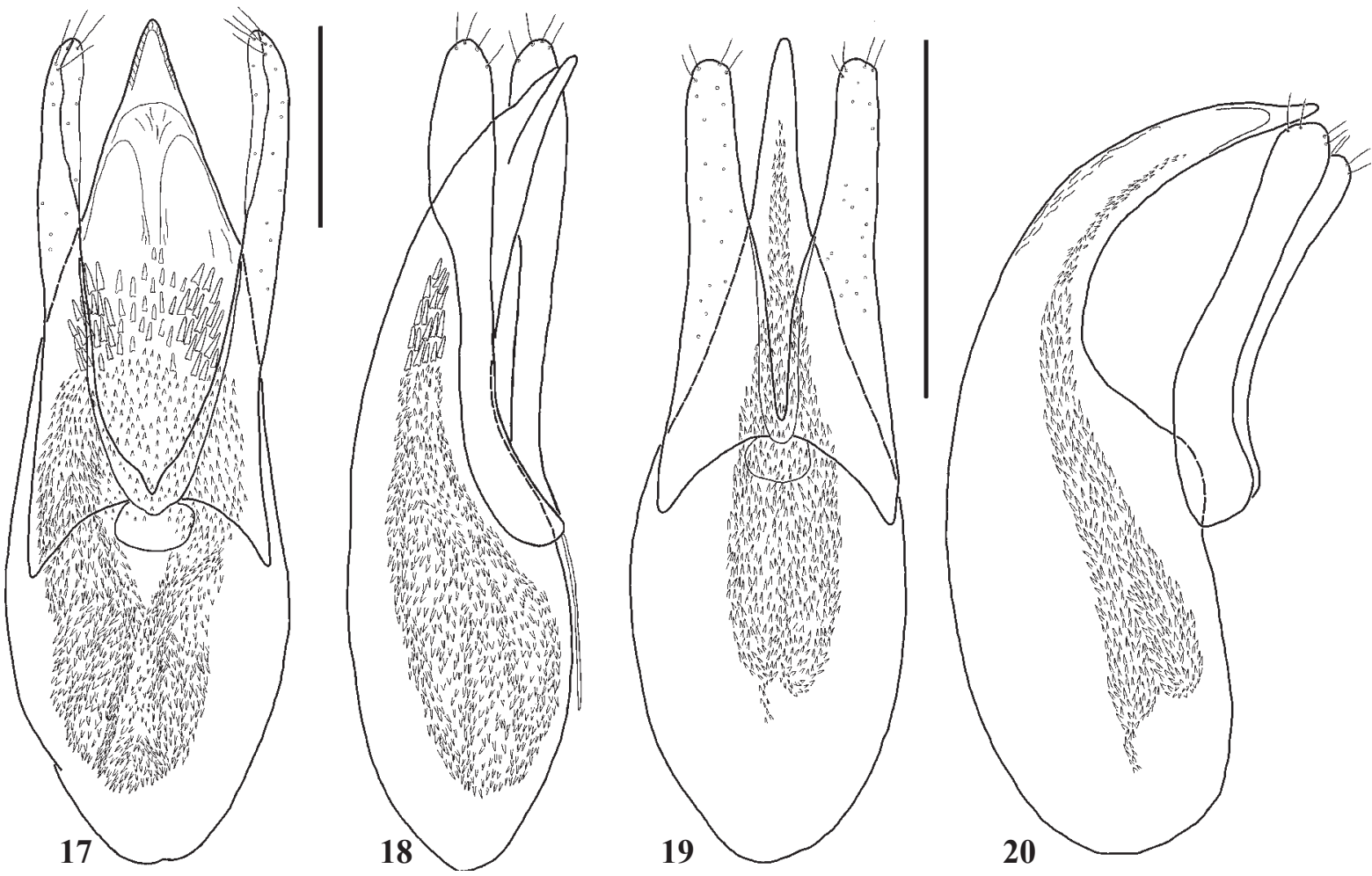

Figs 17-20. Aedeagus of Lesteva spp.: 17-18 - L. nipponica Watanabe; 19-20 - L. plagiata Sharp; 17, 19 — ventral view; 18, 20 lateral view. Scale bars: $0.1 \mathrm{~mm}$.

Рис. 17-20. Эдеагус Lesteva spp.: 17-18 - L. nipponica Watanabe; 19-20 - L. plagiata Sharp; 17, 19 - снизу; 18, 20 — сбоку. Масштаб: 0.1 мм. 

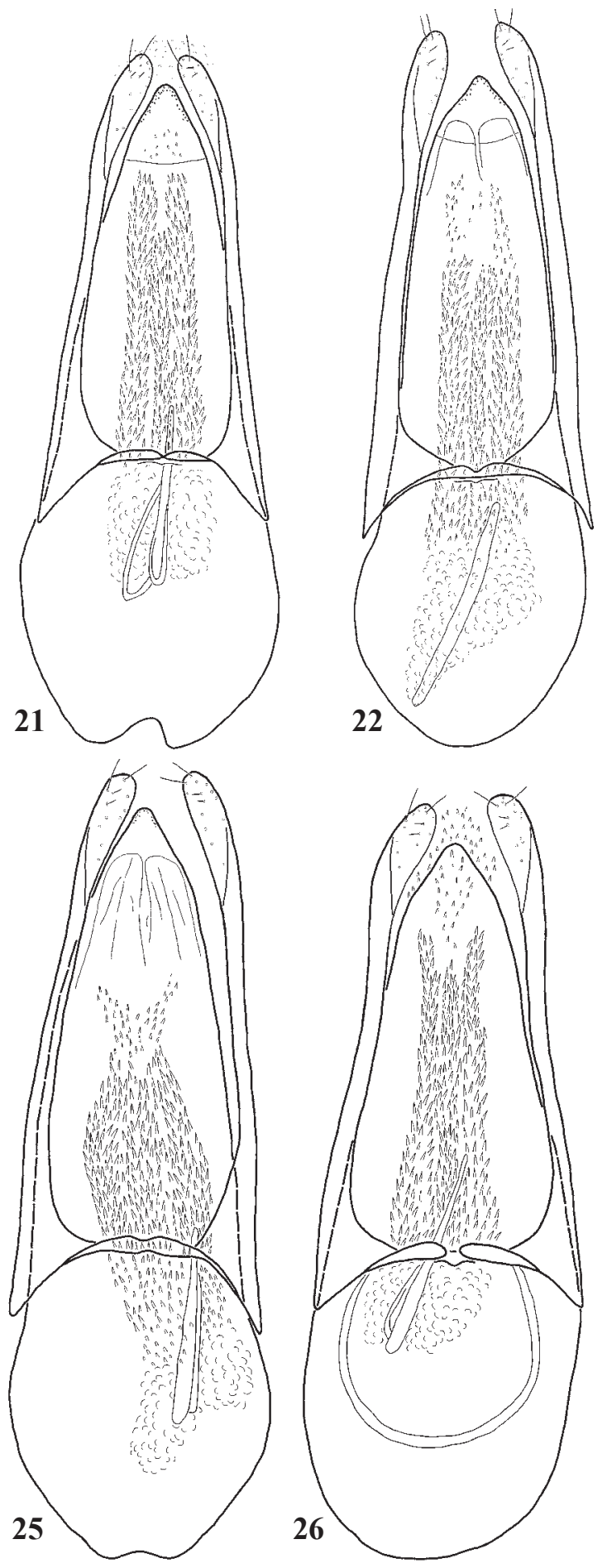

Figs 21-26. Aedeagus of Liophilydrodes puncticollis (Nakane et Sawada), ventral view: 21 - Kunashir Island, Filatov River; $22-$ Kunashir Island, Golovnin River; 23 - Kunashir Island, Nelyudimyi Cape; 24 - Sakhalin Island, Peskovka River; 25 - Sakhalin Island, Plastunka River; 26 (with sketched basal pieces of aedeagus) Khabarovsk Territory, Sikhote-Alin Mt. range. Scale bar: $0.1 \mathrm{~mm}$.

Рис. 21-26. Эдеагус Liophilydrodes puncticollis (Nakane et Sawada), снизу: 21 - о-в Кунашир, р. Филатова; 22 - о-в Кунашир, р. Головнина; 23 - о-в Кунашир, мыс Нелюдимый; 24 - о-в Сахалин, р. Песковка; 25 - о-в Сахалин, р. Пластунка; 26 (с нарисованными базальными частями эдеагуса) Хабаровский край, хр. Сихотэ-Алинь. Масштаб: 0.1 мм.
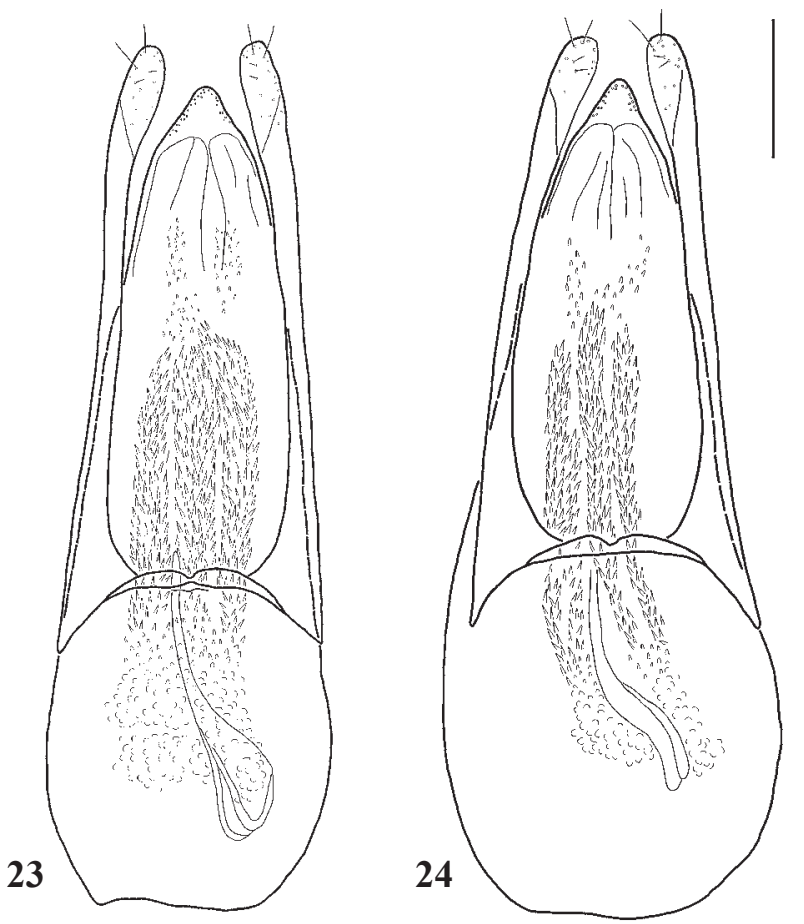

flow of Zolotaya River, 25.07.2013, K.V. Makarov leg. (MSPU); 1 $\sigma^{\top}, 2$ 우: middle flow of Zolotaya River, 15.08.2013, K.V. Makarov, Yu.N. Sundukov leg. (CS, MSPU); 1 T': right side of valley of Severyanka River, 28.07.2013, K.V. Makarov leg. (MSPU); 1 ; valley of Tyatina River, 1-2 km upper of mouth, 07-10.06.2016, Yu.N. Sundukov leg. (CS); 2 Oo: lower flow of Filatov River, 2527.06.2013, Yu.N. Sundukov (CS); 1 ○', 1 \%: same data, 2931.07.2014, (MSPU); $2 \sigma^{7} \sigma^{7}, 1$ : Filatovskiy kordon, 07.09.2009, A.S. Prosvirov (CS); $1 \sigma^{7}$ : middle flow of Serebryanka River, valley, $44^{\circ} 2^{\prime} 53^{\prime \prime N} 145^{\circ} 51^{\prime} 28^{\prime \prime} \mathrm{E}, 18.08 .2017$, K.V. Makarov leg. (MSPU); $1 \sigma^{7}$ : 6 km SW Yuzhno-Kurilsk, Kislaya River, 19.06.1990, K.V. Makarov leg. (ZMM); $2 \sigma^{7} \sigma^{7}$ : env. Mendeleevo, 19.09.2009, I.V. Melnik leg. (CS); $1 \sigma^{7}$ : NW bank of Peschanoye Lake, 07.08.2011, I.V. Melnik leg. (CS); 2 oo: Alyokhino, 11-14.09.2014, Yu.N. Sundukov leg. (CS); 2 +o: lower flow of Andreeva River, 0508.08.2014, Yu.N. Sundukov leg. (MSPU); $2 \bigcirc^{\top} \sigma^{7}, 1$ ㅇ: Okhotskoye natural boundary, 23.07.2011, K.V. Makarov leg. (CS, MSPU); 1 $\mathrm{O}^{7}, 5$ 우: bank of Goryacheye Lake, 22.07.2011, K.V. Makarov leg. (CS, MSPU); 1 : caldera of Golovnin Volcano, environs of Ozy-

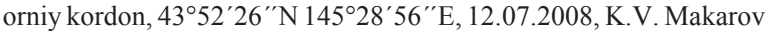
leg. (MSPU); $1 \bigcirc^{7}$ : same data, Goryacheye Lake (except of eastern

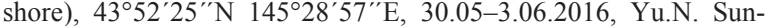
dukov, L.A. Sundukova leg. (MSPU); $1 \sigma^{\text {': }}$ same data and Lake, perennial grasses, 26.09.2012, K. Gongalskiy leg. (CS); $1 \sigma^{7}$ : caldera of Golovnin Volcano, 04-07.2016, Yu.N. Sundukov leg. (CS); 2 $\sigma^{\top} \sigma^{\top}, 4$ 우: same data, 07-09.06.2013, (CS, MSPU); 8 О $\sigma^{\top}, 8$ 우: Ozyorniy kordon, 21-28.07.2011, S.A. Kurbatov leg. (CS, RCPQ); $2 \sigma^{7} \sigma^{7}, 6$ O+: same data, 23-24.04.2018, Yu.N. Sundukov leg. (CS, MSPU); 2 우: Ozyornaya River, 10-11.09.2015, Yu.N. Sundukov leg. (CS).

ADDITIONAL MATERIAL. RUSSIA: $2 \sigma^{\top} \sigma^{\top}, 1$ \% : Khabarovsk Territory, northern Sikhote-Alin, central part of Bolshoy Yan Ridge (western macroslope), $\mathrm{h}=1100-1200 \mathrm{~m}$, bank of stream in coniferous forest, 27-28.07.1997, D.E. Lomakin leg. (ZMUC); $2 \sigma^{7} \sigma^{7}, 1$ 웅 Sakhalin, southern part, Tretya Pad', valley of Plastunka River (Plastushka), $46^{\circ} 42.04^{\prime} \mathrm{N} 142^{\circ} 45.44^{\prime}$ E. 09-12.07.2003, I.V. Melnik leg. (CS, MSPU); $2 \sigma^{7} \sigma^{7}, 1$ : Sakhalin, Krilyon Isthmus, Shebunino, $45^{\circ} 24.59^{\prime} \mathrm{N} 141^{\circ} 51.53^{\prime} \mathrm{E}, 09-18.06 .2003$, I.V. Melnik leg. (CS, MSPU); $3 \sigma^{7} \sigma^{7}, 7$ 우: Sakhalin, $14 \mathrm{~km}$ WNW Lesnoye, valley of Peskovka River, 46 ${ }^{\circ} 57.33^{\prime} \mathrm{N} 142^{\circ} 54.34^{\prime} \mathrm{E}, 23-30.06 .2003$, I.V. Melnik leg. (CS, MSPU); $1 \mathrm{O}^{7}$ : Sakhalin, $10 \mathrm{~km}$ from Bykovo, Naiba River, 14-23.08.1991, V.V. Zherikhin, K.Yu. Eskov, V.G. Gratshev leg. (ZMM); 1 O : Sakhalin, valley of Tym' River, $50^{\circ} 40.08^{\prime} \mathrm{N} 143^{\circ} 05.06^{\prime} \mathrm{E}$, 
04-05.07.2003, I.V. Melnik leg. (CS); 2 우: Shikotan Island, Tserkovnaya Bay, 12-15.08.2016, Yu.N. Sundukov leg. (CS).

DISTRIBUTION. Russia: Khabarovsk Territory, Sakhalin, Kurile Islands (Iturup, Kunashir: Map 9, Shikotan). Japan (Hokkaido).

BIONOMICS. This is a very common species in Kunashir Island. Specimens were collected on stony and occasionally on sandy banks of streams and rivers, and sometimes near thermal zones (Figs 9-12). It was found in a stony shore in the caldera of Goryacheye Lake (Fig. 9).

REMARKS. Liophilydrodes puncticollis has a relatively variably shaped pronotum with fine to moderately coarse punctation, indistinct to distinct microsculpture between punctures both on head and pronotum, and markedly transverse or somewhat elongate elytra. Habitus as in Fig. 6. The width and shape of the apical portion of the median lobe and parameres are subject to little variation (Figs 21-26). The internal sac of the genus Liophilydrodes is here presented for the first time: L. puncticollis has a relatively complex and variable shape of it (compare Figs 22, 24-26 and Figs 21, 23). Liophilydrodes puncticollis is recorded from Kunashir and Shikotan islands, and from the mainland Asia (Russia, Khabarovsk Territory) for the first time.

In general, the genus Liophilydrodes is in need of a taxonomic revision and comparative morphological study of the variability of the body and aedeagus. This could eventually lead to new synonymies of some names from Japan, which are apparently more widely distributed. It is worth noting that L. subtilis iturupensis, described based on one female from Iturup Island [Lafer, 2004], in some details of the body differs from two syntypes of Anthophagus subtilis Sharp, 1889, deposited in BMNH and known to the senior author. However, a study of additional material from the type locality and other adjacent islands is required for the clarification of its taxonomic status.
Mannerheimia yasudai Watanabe, 1990

Fig. 16, 27-28; Map 10.

Mannerheimia yasudai Watanabe, 1990: 86 (type locality: Japan, Hokkaido, Mt. Kurodake on Daisetsu Mts.)

MATERIAL. 1 +: Saratovskaya River - Tyatina River, shore near Ploskogornyi Cape, $44^{\circ} 15^{\prime} 56^{\prime \prime} \mathrm{N} 146^{\circ} 08^{\prime} 37^{\prime \prime} \mathrm{E}, 21-$

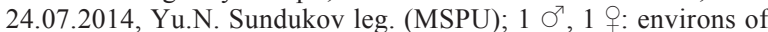
Tretyakovo, litter, near stream, 18-21.07.1990, S.A. Kurbatov leg. (CS); 1 क: Ozyornyi kordon, 21-28.07.2011, S.A. Kurbatov leg. (RCPQ).

DISTRIBUTION. Russia: southern Kurile Islands (Kunashir: Map 10). — Japan (Hokkaido).

BIONOMICS. Specimens were collected on the banks of streams and rivers with cold water, more frequently in the thermal zone (Fig. 16).

REMARKS. In the present study we provide figures of the aedeagus (Figs 27-28), including the internal sac. The species is recorded from Russia and Kunashir for the first time.

\section{Olophrum sp.}

MATERIAL. 1 o': Mendeleevo, 11.07.1985, S.A. Kurbatov (temporarily in CS, to be eventually deposited in ZMM).

REMARKS. The present male apparently belongs to a new species related to $O$. simplex Sharp, 1874 from Japan, but additional material is required to confirm this.

\section{Coryphiini Jakobson, 1908}

Archaeoboreaphilus miyamorii (Watanabe, 1988)

Figs 7, 12, 31-37; Map 11.

Coryphium miyamorii Watanabe, 1988: 49 (type locality: Japan, Hokkaido, Horobetsu-kôzan, Iburi); Watanabe, 1990: 359; Archaeoboreaphilus mivamorii: Zerche, 1990: 163

MATERIAL. 1 ㅇ: Stream ab. $1 \mathrm{~km}$ SE Dokuchaevo (nonresidential), $44^{\circ} 30^{\prime} 21^{\prime \prime} \mathrm{N} 146^{\circ} 10^{\prime} 01^{\prime \prime} \mathrm{E}, \mathrm{h}=100 \mathrm{~m}, 01.08 .2013$, K.V.
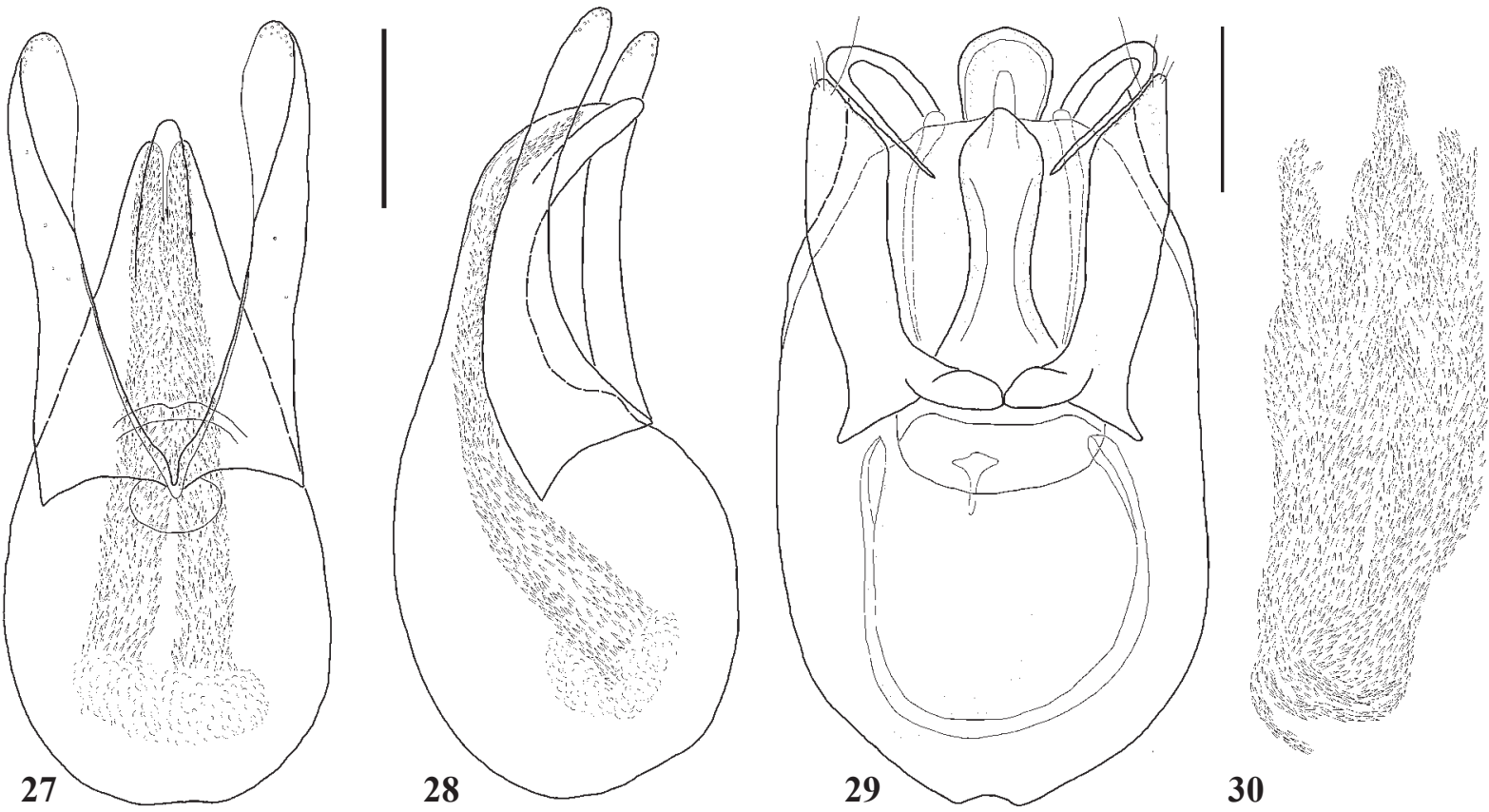

Figs 27-30. Aedeagus: 27-28 - Mannerheimia yasudai Watanabe; 29-30 - Acrolocha miyamorii Watanabe; 27,29 - ventral view (outline of internal sac shown as point line), 28 - lateral view, 30 - internal sac, ventral view. Scale bar: $0.1 \mathrm{~mm}$.

Рис. 27-30. Эдеагус: 27-28 - Mannerheimia yasudai Watanabe; 29-30 - Acrolocha miyamorii Watanabe; 27, 29 - снизу (контур внутреннего мешка показан в виде точечной линии); 28 - сбоку; 30 - внутренний мешок, вентральная сторона. Масштаб: 0.1 мм. 
Makarov leg. (MSPU); $10^{7}$ : Stolbovskoy [Zmeiny] Stream above springs, 21.07.2013, K.V. Makarov leg. (CS); 1 \%: Ozyornyi kordon, 21-28.07.2011, S.A. Kurbatov leg. (ZMM).

DISTRIBUTION. Russia: southern Kurile Islands (Kunashir: Map 11). — Japan (Hokkaido, Honshu).

BIONOMICS. The specimens were collected on the banks of cold streams near thermal springs (Fig. 12).

REMARKS. Coryphium miyamorii was described based on numerous specimens from Hokkaido and Honshu [Watanabe, 1988]. Zerche [1990] studied only the holotype and did not provided figures of mouthparts and apical abdominal sclerites, while Watanabe [1988, 1990] published figures of the aedeagus in two projections and the ventral view of the median lobe. Habitus as in Fig. 7. In the present paper, we present the following figures: aedeagus with somewhat deformed basal portion (Fig. 37), labrum (Fig. 31), maxillary palpus (Fig. 32), labium and mentum (Fig. 33), male sternite IX (Fig. 34), male tergite VIII (Fig. 35) and male sternite VIII (Fig. 36). This species is recorded for Russia and Kunashir for the first time.

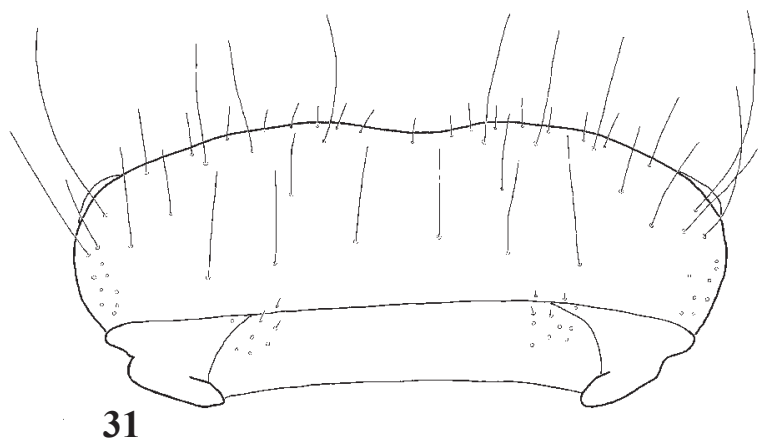

31
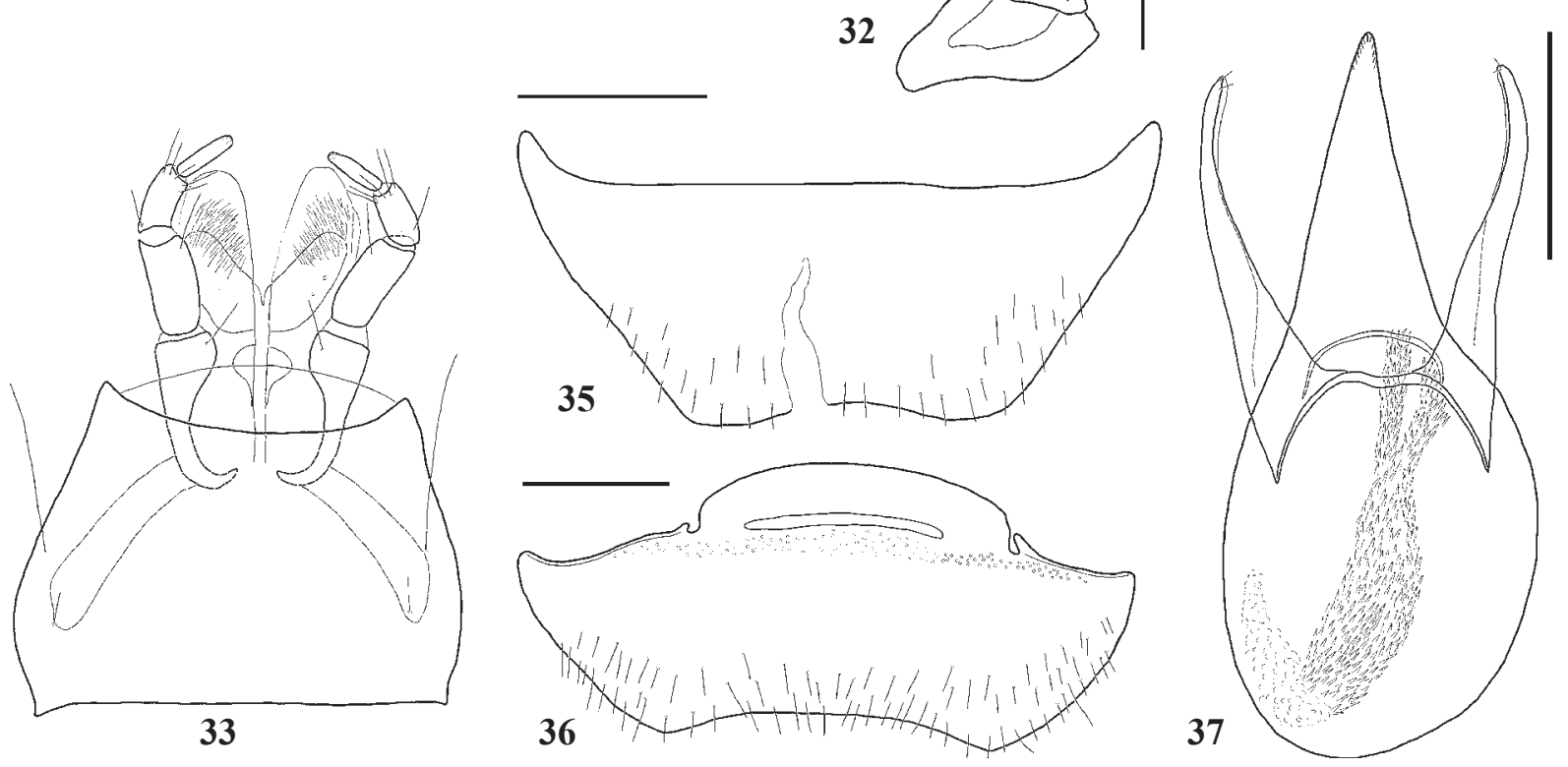

Figs 31-37. Archaeoboreaphilus miyamorii (Watanabe): 31 - labrum, dorsal view; 32 - left maxillary palpus, dorsal view; 33 labium and mentum, ventral view; 34 - male sternite IX; 35 - male tergite VIII; 36 - male sternite VIII; 37 - aedeagus, ventral view. Scale bars: $0.1 \mathrm{~mm}$.

Рис. 31-37. Archaeoboreaphilus miyamorii (Watanabe): 31 - верхняя губа, сверху; 32 - левый челюстной щупик, сверху; 33нижняя губа и подбородок, снизу; 34 - IX стернит самца, снизу; 35 - VIII тергит самца, сверху; 36 - VIII стернит самца, снизу; 37 - эдеагус, сбоку. Масштаб: 0.1 мм. 
MATERIAL. 1 : lower flow of Severyanka River, 44²0'17'’ N $146^{\circ} 00^{\prime} 51^{\prime \prime} \mathrm{E}, 05-08.06 .2014$, Yu.N. Sundukov leg. (MSPU); $1 \sigma^{7}, 1$

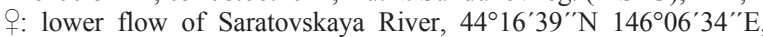
window traps, 12-18.07.2014, Yu.N. Sundukov leg. (CS); 1 : same data, 4415'46"N 14606'13"E, 22-25.07.2014, Yu.N. Sundukov, L.A. Sundukova leg. (MSPU); $1 \sigma^{7}$ : environs of Andreevskiy kordon, 07.07.2014, A.S. Prosvirov leg. (CS).

DISTRIBUTION. Russia: southern Kurile Islands (Iturup, Kunashir: Map 13). — Japan (Hokkaido).

BIONOMICS. Detailed ecological data are not available.

REMARKS. In the present study we provide figures of the aedeagus (Fig. 29) and the internal sac (Fig. 30). This species is recorded from Kunashir for the first time.

\section{Omalium sp.}

MATERIAL. $1 \bigcirc^{7}$ : Between Saratovskaya and Tyatina rivers, $44^{\circ} 16^{\prime} 28^{\prime \prime} N 146^{\circ} 07^{\prime} 09^{\prime \prime}$ E, 5-8.07.2014,Yu.N. Sundukov, L.A. Sundukova leg. (CS).

REMARKS. The studied specimen possibly belongs to a new species of the rivulare group sensu Zanetti [1987]. East Palaearctic species of this group have not been revised.

$$
\begin{gathered}
\text { Phloeostiba plana (Paykull, 1792) } \\
\text { Map 14. }
\end{gathered}
$$

The synonymy and other references see in Herman [2001] and Schülke \& Smetana [2015]

MATERIAL. 1 : valley of Tyatina River, 1-2 km up-stream of mouth, 7-10.06.2016, Yu.N. Sundukov leg. (CS); $1 \sigma^{7}$ : Alyokhina Cape, 02.08.2011, Yu.N. Sundukov leg. (MSPU); $10^{7}, 19$ : bank of

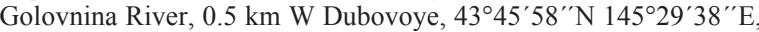
21.08.2017, K.V. Makarov, Yu.N. Sundukov leg. (CS).

ADDITIONAL MATERIAL. RUSSIA: $1 \sigma^{7}, 2$ OO: Maritime Province, Lazovskiy Nature Reserve, Zvyozdochka kordon, 1421.05.2009, S.A. Kurbatov leg. (RCPQ).

DISTRIBUTION. Trans-Palaearctic species.

BIONOMICS. Specimens were collected under bark of coniferous trees, predominantly Abies.

REMARKS. This species is recorded from Kunashir (Map 14) for the first time.

\section{Phloeonomus orientalis Smetana, 1981 Map 15.}

Phloeonomus (Phloeonomus) orientalis Smetana, 1981: 78 (type locality: Russia, Kunashir, Mendeleevo); Phloeonomus sp.: Shavrin, Gildenkov, 2009: 122.

MATERIAL. 1 ○’, 1 : ‘10.9.[19]72 Кунашир | Менделеево | короедн. [?] пихта | в ходах коро- | едов. доп. к N9. | Потоцкая [10.9.72 Kunashir, Mendeleevo [...] firs, in galleries of bark beetles [...] [V.A.] Pototskaja]', 'Phloeonomus | orientalis Smet. | Smetana

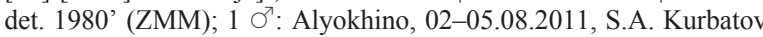
leg. (CS).

ADDITIONAL MATERIAL. RUSSIA: 1 : : Maritime Province, Lazovskiy Nature Reserve, Zvyozdochka kordon, 14-21.05.2009, S.A. Kurbatov (CS); 1 क: same Reserve, Petrov Island, 26-29.05. 2000, Yu.N. Sundukov (CS).

DISTRIBUTION. Russia: Maritime Province, southern Kurile Islands (Kunashir: Map 15).

BIONOMICS. The species inhabit bark of logs and trees. Detailed ecological data for Kunashir Island are not available. REMARKS. This species is recorded from mainland Asia (Russia, Maritime Province) for the first time.

\section{Phloeonomus pusillus (Gravenhorst, 1806) Map 16.}

The synonymy and other references see in Herman [2001] and Schülke \& Smetana [2015].

MATERIAL. 1 ○', 2 क्ष: Ozyorniy kordon, 21-28.07.2011, S.A. Kurbatov (CS).

DISTRIBUTION. Holarctic species.

BIONOMICS. See data for the species above.
REMARKS. This species is recorded from Kunashir for the first time.

Eusphalerini Hatch, 1957

Eusphalerum sp.pr. hidakanum Watanabe, 1990 Map 17.

Eusphalerum prope hidakanum Watanabe, 1990: Zanetti, 1993

MATERIAL: $2 \sigma^{7} \sigma^{7}, 1$ : Ivanovskiy Cape, Grozovoye natural boundary, $43^{\circ} 50^{\prime} 28^{\prime \prime} \mathrm{N} 145^{\circ} 24^{\prime} 29^{\prime \prime} \mathrm{E}, 02-04.06 .2013$, Yu.N. Sundukov, L.A. Sundukova leg. (CS); 1 O: Ivanovskiy Cape, 0821.09.2014, Yu.N. Sundukov (CS); 1 q: same data, Grozovoye, $43^{\circ} 50^{\prime} 28^{\prime \prime} \mathrm{N} 145^{\circ} 24^{\prime} 29^{\prime \prime}$ E, 08-09.07.2013, Yu.N. Sundukov, L.A. Sundukova leg. (CS).

REMARKS. The aedeagus of the examined males is similar to the illustrations provided in Figs 27-28 in Zanetti [1993]. The shape of apical portion of the median lobe resembles that of $E$. hidakanum Watanabe, 1990, which was described from Hokkaido (Mt. Daisetsu), Japan. A study of type material is necessary for reliable identification.

\section{Eusphalerum sp.}

MATERIAL. 1 ㅇ: Lower flow of Severyanka River, $44^{\circ} 19^{\prime} 58^{\prime \prime} \mathrm{N}$ $146^{\circ} 01^{\prime} 15^{\prime \prime} \mathrm{E}, 10-14.06 .2014$, Yu.N. Sundukov leg. (CS).

\section{Discussion}

In the present paper, 19 species of rove beetles of the subfamily Omaliinae belonging to 14 genera in four tribes are recorded: Anthophagini (7 genera: 10 species), Coryphiini (2 genera: 2 species), Omaliini (4 genera: 5 species) and Eusphalerini (1 genus: 2 species). Before this study, only nine species of this subfamily were known [Filatova, Lafer, 1978; Lafer, 1989, 2004; Zanetti, 1993; Shibata et al., 2013; Shavrin, 2018] from Kuriles, five of which were recorded from $\mathrm{Ku}-$ nashir Island. However, records of some these species require further clarification (see Introduction). Except for four species, which belong to unrevised and possibly new taxa, the material was identified to species level. The list of species from Kunashir is preliminary and in future can be significantly increased by records of species of the genera Acidota Stephens, 1829, Geodromicus Redtenbacher, 1857, Lesteva Latreille, 1797, Olophrum Erichson, 1839, Boreaphilus Sahlberg, 1832, Eusphalerum Kraatz, 1857, and several others. However, in comparison with what is known about the Omaliinae fauna of adjacent regions, this subfamily of $\mathrm{Ku}$ nashir Island is currently relatively well studied. For example, 15 species have been recorded from Kamchatka [Ryabukhin, 1991, 1999, 2008, 2010], five species from Commander Islands [Sazhnev, 2018; Ryabukhin, 1999], 11 species from Sakhalin Island [Molodova, 1973; Lafer, 1989; Zanetti, 1993; Shavrin, Berlov, 1999; Herman, 2001; Zerche, 1993, 2003; Shavrin, 2018], three species from Iturup Island [Filatova, Lafer, 1978; Lafer, 2004] and 40 species from Hokkaido, Japan [Shibata et al., 2013]. Most of the recorded taxa from the studied island are Eastern Palaearctic species with insufficiently known limits of the distribution, and only two species are widely distributed: the trans-Palaearctic Phloeostiba plana and the Holarctic Phloeonomus pusillus. The present study specifies the distributions of 
these two widespread species in the islands of the Fast East and additionally those of several eastern species which were previously known only from Japan (Acidota daisetsuzana, Lesteva nipponica, Mannerheimia yasudai, Archaeoboreaphilus miyamorii, Eudectus rufulus) or were not recorded from the mainland part of Eurasia (Liophilydrodes puncticollis and Phloeonomus orientalis). Most of the taxa of Omaliinae in the present study represent hygrophilous species which are common on the banks of streams and rivers. Some species are xylophilous and can be found under bark of deciduous and coniferous trees (Phloeonomus), and unidentified members of the genus Eusphalerum are anthophilous species which are attracted to flowers, mostly of flowering bushes.

The present-day fauna and flora of the southern islands of the Kuril Archipelago are characterized by a high species diversity and low level of endemism. The can thus be regarded as an example of "non-relic" formation of the biome [Pietsch et al., 2003]. It is assumed that migration events from Hokkaido played a main role in the development of the fauna of the southern Kurils. It makes sense, since, throughout almost the entire Pleistocene, Kunashir like all other southern Kurile Islands, were associated with Hokkaido and Sakhalin [Korotkii, 1985; Bogatov et al., 2006]. In addition, during the Holocene the successive transgressions and regressions of the sea at least twice created a land link beween Kunashir and Hokkaido [Razjigaeva et al., 2013]. The question remains how the unity of these islands has promoted the free exchange of the fauna. On the one hand, these periods coincide with the cooling, as a result of which Sakhalin completely and Hokkaido partially found themselves in a permafrost zone [Igarashi, Zharov, 2011], which significantly limited the migration of beetles. On the other hand, the palynological spectra suggest that the ancient land connecting Kunashir, the Lesser Kuriles, and Hokkaido was covered with forests, including elements of the nemoral vegetation [Razjigaeva et al., 2008, 2011]. It is likely that the degree of the isolation of islands and types of the vegetation accompained by the extinction of some taxa were influenced by the land area transformations during glacial-interglacial cycles in the Pleistocene and the Holocene. Moreover, in conditions of partial isolation, the island fauna was influenced to a much greater degree by the glaciation and catastrophic events, in this case by the activities of volcanic eruptions and tsunamis [Razjigaeva et al., 2011]. Although throughout the Late Pleistocene and Holocene, signs of the surface glaciation were not observed for the southern Kuriles [traces of fragmented glaciation of the Kuriles do not extend to the south of the northern part of Iturup Island - Atlas of Kurile Islands, 2009], the modern faunal composition of each of the islands depended on the presence of hydrothermal fields in the refugia of the volcanic activity zones [Razjigaeva, Ganzei, 2004]. An example of this type of distribution could be Bembidion negrei Habu, 1958 (Carabidae), which is known from thermal zones of Hokkaido and Honshu and registered only in the caldera of Golovnin Volcano in Kunashir [Morita, 2010]. The impact of tsunamis on the fauna of Kunashir is weakened by its relief, and a significant impact can be assumed only for the southern part of the island, in Sernovodskiy and Yuzhnokurilskiy isthmuses. Lowaltitude islands of the Lesser Kuril Chain are much more vulnerable in this respect. At least for Polonskiy and Zelyonyi islands, it was shown that they were completely or partly flooded by tsunamis several times in the Holocene [Razjigaeva et al., 2008]. It is notable that species of Omaliinae were not found on these islands. Major explosive volcanic eruptions on Kunashir Island took place in the late Pleistocene [Razjigaeva et al., 2011]. As a result, the main stratovolcanoes and calderas of the island were formed, and the pyroclastic flows of Golovnin and Mendeleev volcanoes created the entire southern part of the island [Bazarova et al., 1998], that is, the formation of the biota in this region is mainly the result of migration processes. In contrast, the Dokuchaev Ridge, which is located in the northern part of the island, was formed by Lower and Middle-Miocene rocks [Furaev, 2013] and two large Smirnova and Rurui volcanoes with little activity in the Pleistocene after the Last Glacial Maximum [Gorshkov, 1967]. At present, these volcanoes are badly damaged [Furaev, 2013]. The intense formation of primary volcanic landscapes in the Holocene was associated with eruptions of the Tyatya Volcano [Razjigaeva, Ganzei, 2004], while the remaining volcanoes remained nearly inactive. Thus, starting from the Pleistocene, the northwestern part of the island with the Dokuchaev Ridge may have served as a refugium. The existence of this refugium for a long time could ensure not only the survival, but also the appearance of endemic beetles [Makarov, Sundukov, 2014]. Geomorphologically, the Dokuchaev mountain range forms a unit with the Shiretoko mountain range of Hokkaido. However, the Staphylinidae fauna of the latter has not been studied in detail, and only the southern regions of Hokkaido are relatively well studied. Additional studies of this region may lead to very interesting results.

Species of Omaliinae are unevenly distributed in Kunashir Island and, in general, not common. Only two species, Liophilydrodes puncticollis and Brathinus oculatus, were found quite often and are relatively evenly distributed in the northern and southern parts of the island. Their distributions are not limited by the hydrothermal zones, and populations can be quite dense. At the same time, L. puncticollis avoids floodplains in the southern part of the island in Sernovodskiy and Yuzhnokurilskiy isthmuses. The remaining species of the subfamily are much less common and only the patterns of distribution of a few species could be assessed. Of particular interest are the distributions of Lesteva nipponica, Mannerheimia yasudai, and Archaeoboreaphilus miyamorii: these species were found on the banks of cold streams in the thermal zones of Rurui, Mendeleev and Golovnin volcanoes. Such a pattern is very much in line with the concepts of thermal zones as surviving habitats [Lelei et al., 2002] during periods of pessima. Considering that 

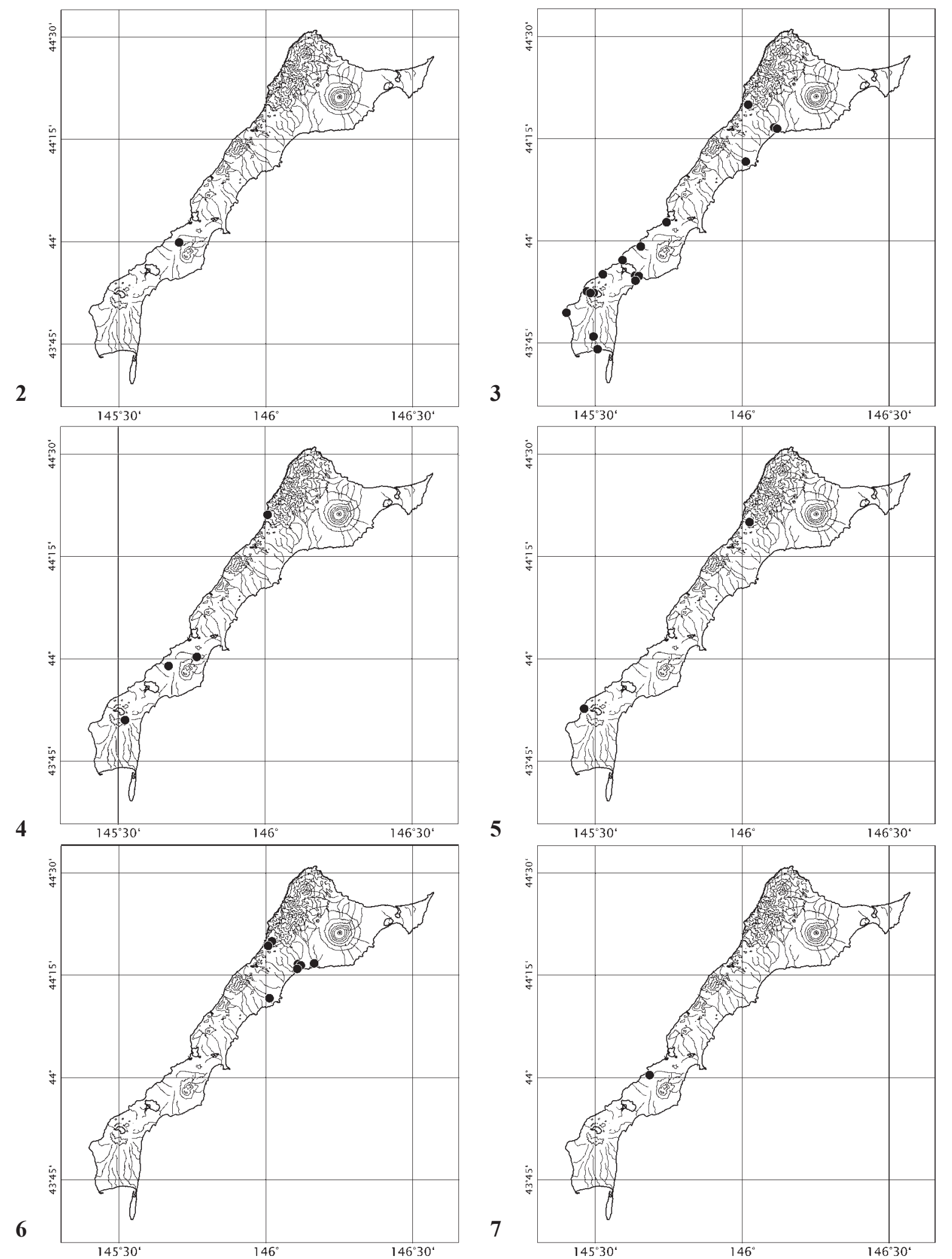

Maps 2-7. Distribution of Omaliinae in Kunashir Island: 2 -Acidota daisetsuzana Watanabe, 1990; 3 - Brathinus oculatus Lewis, 1886; 4 - Geodromicus iburinus (Watanabe, 1990); 5 - Geodromicus lestevoides (Sharp, 1889); 6 - Geodromicus nipponensis (Watanabe, 1990); 7 - Lesteva nipponica Watanabe, 1990.

Карты 2-7. Распространение Omaliinae на острове Кунашир: 2 - Acidota daisetsuzana Watanabe, 1990; 3 - Brathinus oculatus Lewis, 1886; 4 - Geodromicus lestevoides (Sharp, 1889); 5 - Geodromicus nipponensis (Watanabe, 1990); 6 — Geodromicus iburinus (Watanabe, 1990); 7 - Lesteva nipponica Watanabe, 1990. 

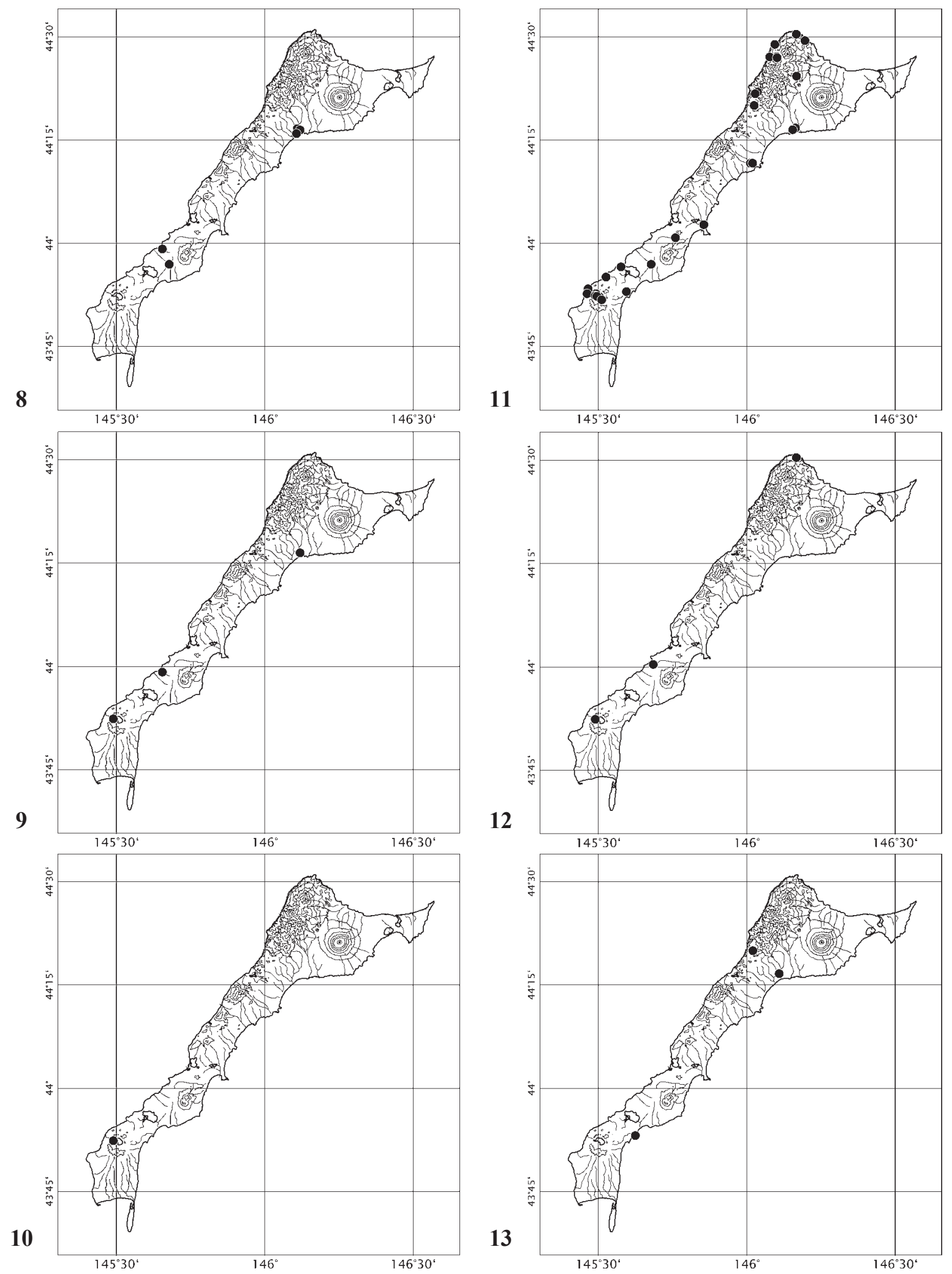

Maps 8-13. Distribution of Omaliinae in Kunashir Island: 8 - Lesteva plagiata Sharp, 1889; 9 - Liophilydrodes puncticollis (Nakane et Sawada, 1956); 10 - Mannerheimia yasudai Watanabe, 1990; 11 - Archaeoboreaphilus miyamorii (Watanabe, 1988); 12 — Eudectus rufulus Weise, 1877; 13 - Acrolocha miyamorii Watanabe, 1990.

Карты 8-13. Распространение Omaliinae на острове Кунашир: 8- Lesteva plagiata Sharp, 1889; 9 - Liophilydrodes puncticollis (Nakane et Sawada, 1956); 10 - Mannerheimia yasudai Watanabe, 1990; 11 - Archaeoboreaphilus miyamorii (Watanabe, 1988); 12 Eudectus rufulus Weise, 1877; 13 - Acrolocha miyamorii Watanabe, 1990. 

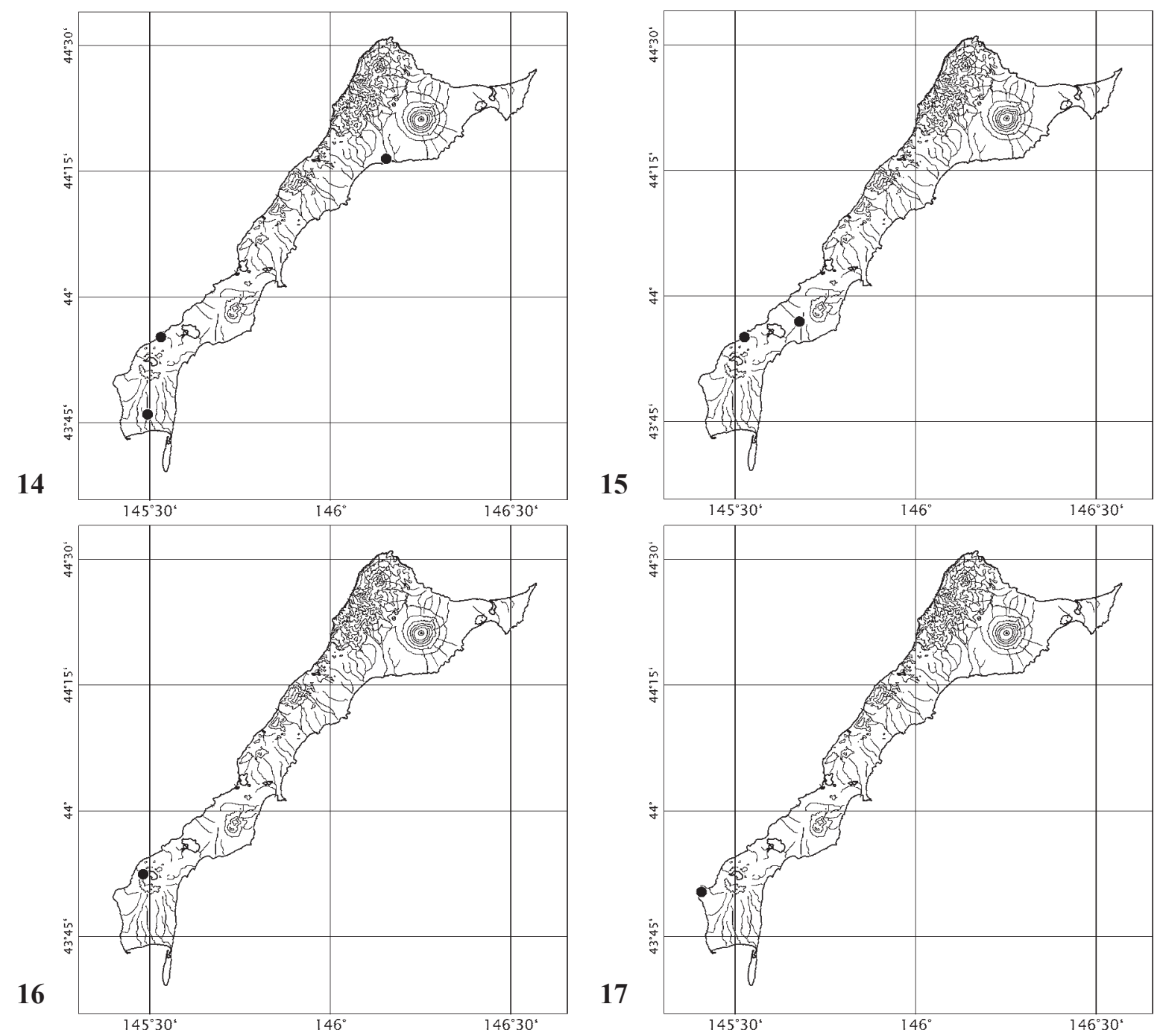

Maps 14-17. Distribution of Omaliinae in Kunashir Island: 14 - Phloeostiba plana (Paykull, 1792); 15 - Phloeonomus orientalis Smetana, 1981; 16 - Phloeonomus pusillus (Gravenhorst, 1806); 17 - Eusphalerum sp.pr. hidakanum Watanabe, 1990.

Карты 14-17. Распространение Omaliinae на острове Кунашир: 14 - Phloeostiba plana (Paykull, 1792); 15 — Phloeonomus orientalis Smetana, 1981; 16 - Phloeonomus pusillus (Gravenhorst, 1806); 17 - Eusphalerum sp.pr. hidakanum Watanabe, 1990.

most of these thermal zones arose as a result of Late Pleistocene eruptions [Razjigaeva, Ganzei, 2004], it is obvious that these sites played the role of refugia during the Last Glacial Maximum. However, for Omaliinae, most of which are strictly temperate species that can exist at low temperatures, the value of such refugia may not be so important. In this sense, the distributions of species of Geodromicus and Lesteva in Kunashir are indicative: G. iburinus and L. nipponica are related with thermal zones (mainly Mendeleev and Golovnin volcanoes), and G. nipponensis and L. plagiata are distibuted much more widely along river banks in the foothills of the Dokuchaev Mt. and Mendeleev Volcano (L. plagiata).

Acknowledgements. We are thank our colleagues for making Omaliinae specimens available for this study: Roger G. Booth (London, UK), Yvonnick Gérard (Bruxelles, Belgium), Andrey V. Matalin, Igor V. Melnik, Alexey A. Gusakov,
Sergey A. Kurbatov, Alexandr S. Prosvirov and Alexandr B. Ryvkin (Moscow, Russia), Harald Schillhammer (Vienna, Austria), Alexey Yu. Solodovnikov (København, Denmark), Yuriy N. Sundukov and Larisa A. Sundukova (Lazo, Russia), Mark G. Volkovitch and Svetlana V. Andreeva (St. Petersburg, Russia). Special thanks are extended to Yuriy N. Sundukov for vast material from the studied island and photographs of localities, and Volker Assing (Hannover, Germany) for correction of the English text of the manuscript.

\section{References}

Atlas of Kurile Islands. 2009. Designe Information-Cartography, Moscow-Vladivostok. 516 pp. [In Russian]

Barkalov V.Yu. 2009. Flora of the Kurile Islands. Vladivostok: Dal'nauka. 468 pp. [In Russian]

Bazarova V.B., Razjigaeva N.G., Grebennikova T.A., Ganzey L.A., Mokhova L.M., Korotky A.M. 1998. Radiocarbon dating of Late Pleistocene-Holocene event on Kunashiri Island, Kurile Islands // Radiocarbon. Vol.40. P.775-780. 
Bogatov V.V., Pietsch T.U., Storozhenko S.Yu., Barkalov V.Yu., Leley A.S., Kholin S.K., Krestov P.V., Kostenko V.A., Makarchenko E.A., Prozorova L.A., Shed'ko S.V. 2006. [Peculiaries of the formation of land and freshwater biota in Sakhalin Island] // Vestnik DVO RAN [Herald of the Far East Department of the Russian Academy of Sciences]. No.2. P.32-47 [in Russian].

Cho Y.B., Lafer G.Sh., Paik J.C., Park, J.K. 2002. Contribution to the staphylinid fauna (Coleoptera, Staphylinidae) of Korea // Korean Journal of Soil Zoology. Vol.7. Nos1-2. P.35-44.

Filatova L.D., Lafer G.Sh. 1978. K izucheniyu fauny staphylinid (Coleoptera, Staphylinidae) ostrova Iturup // Aktual'nyie voprosy okhrany prirody na Dal'nem Vostoke. DVNC AN SSSR, Vladivostok. P.148-153 [in Russian].

Furaev E.A. 2013. [Geochemistry of Kunashir Island Landscapes (Kurile Islands)]. Moscow: Prometei. 180 pp. [In Russian]

Gorshkov K.S. 1967. [Volcanism of the Kuril Island Arc]. Moscow: Nauka. 183 pp. [In Russian]

Hammond P.M. 1971. The systematic position of Brathinus Leconte and Camioleum Lewis (Coleoptera: Staphylinidae) // Journal of Entomology (B). Vol.40. No.1. P.63-70.

Herman L.H. 2001. Catalogue of the Staphylinidae (Insecta: Coleoptera). 1758 to the end of the second millenium // Bulletin of the American Museum of Natural History. Vol.265. Nos1-7. P.1-4218.

Igarashi Y., Zharov A.E. 2011. Climate and vegetation change during the Late Pleistocene and Early Holocene in Sakhalin and Hokkaido, Northeast Asia. // Quaternary International. Vol.237. Nos1-2. P.24-31.

Kim T.-K., Ahn K.-J. 2011. Taxonomy of Korean Lesteva with a decription of a new species (Coleotera: Staphylinidae) // Florida Entomologist. Vol.94. No.1. P.28-34

Kim T.-K., Ahn K.-J. 2014. Taxonomy of the genus Eudectus Redtenbacher (Coleoptera: Staphylinidae: Omaliinae) in Korea // Korean Journal of Applied Entomology. Vol.53. No.2. P.171-175.

Krivolutskaja G.O. 1973. [Entomofauna of the Kurile Islands. Principal features and origin]. Leningrad: Nauka. 316 pp. [In Russian]

Kryzhanovskiy O.L., Tichomirova A.L., Filatova L.D. 1973. [Staphylinids (Coleoptera, Staphylinidae) of southern Primorie] // Ghilarov M.S. (ed.). Ekologia pochvennykh bespozvonochnykh. Moscow: Nauka. P.144-173 [in Russian].

Korotkii A.M. 1985. Quaternary sea-level fluctuations on the northwestern shelf of the Japan Sea // Journal of Coastal Research No.1. P.293-298.

Lafer G.Sh. 1989. [Sem. Brathinidae] // P.A. Lehr (ed.). Opredelitel nasekomykh Dalnego Vostoka SSSR. T.3. Zhestkokrylye, ili zhuki. Part 1. Leningrad: Nauka. P.346 [in Russian].

Lafer G.Sh. 2004. [New subspecies Liophilydrodes subtilis iturupensis subsp. n. (Coleoptera, Staphylinidae: Omaliinae) from Kurile Islands] // A. I. Kurentsov's Annual Memorial Meetings. Vol.15. P.89-93 [in Russian].

Lelei A.S., Storozhenko S.Yu., Kholin S.K. 2002. [Insects (Insecta)] // Rastitelnyi i zhivotnyi mir Kurilskikh ostrovov. Vladivostok: Dalnaika. P.96-108 [in Russian].

Lewis G. 1886. A new species of Brathinus (Silphidae) // The Entomologist's Monthly Magazine. Vol. 23. P.85.

Makarov A.V. 2019. Insect mapping software // https://github.com/ loafer-mka/bmpgen2

Makarov K.V., Sundukov Yu.N. 2014. Bembidion (?Nipponobembidion) ruruy sp. n., a new brachypterous ground beetle (Coleoptera, Carabidae) from Kunashir Island, Kuriles, Russia // ZooKeys. No.463. P.75-93. doi: 10.3897/zookeys.463.8504.

Molodova L.P. 1973. [Fauna of soil invertebrates of Southern Sakhalin] // M.S. Ghilarov (ed.) Ekologia pochvennykh bespozvonochnykh. Moscow: Nauka. P.60-74 [in Russian].

Morita S. 2010. Notes on the Bembidiinae (Coleoptera, Carabidae) of Japan XXIII. Bembidion (Ocydromus) negrei Habu and its new relatives, found in the habitats of hot springs // Elytra (Tokyo). Vol.16. No.1. P.13-21.

Nakane T. 1961. New or little-known Coleoptera from Japan and its adjacent regions. XV // Fragmenta Coleopterologica. Vol.1961. No.1. P.1-6.
Nakane T. 1963. Staphylinidae // T. Nakane, K. Ohbayashi, S. Nomura, Y. Kurosawa (eds.). Iconographia Insectorum Japonicorum, Colore naturali edita. 2. Tokyo: Hokuryukan. P.81-100 [in Japanese].

Nakane T., Sawada K. 1956. On the genus Philydrodes Bernhauer in Japan with descriptions of a new subgenus and several new species // The Scientific Reports of the Saikyo University (A). Vol.2. P.179-186.

Naomi S.-I. 1989. Staphylinidae // Y. Hirashima (ed.). A check list of Japanese insects, compiled by Y. Hirashima. Entomological Laboratory, Faculty of Agriculture, Kyûshû University, Fukuoka. P. 256-287.

Naomi S.-I., Kuranishi R.B., Saito A., Maruyama M. 2000. A list of the family Staphylinidae (Insecta: Coleoptera) collected during the Biological Expedition to the Kamchatka Peninsula and the North Kurile Islands in 1996 and 1997 // Natural History Research. Special Issue 7. P.101-111.

Pietsch Th.W., Bogatov V.V., Amaoka K., Zhuravlev Yu.N., Barkalov V.Yu., Gage S., Takahashi H., Lelej A.S., Storozhenko S.Yu., Minakawa N., Bennett D.J., Anderson T.R., Ohara M., Prozorova L.A., Kuwahara Y., Kholin S.K., Yabe M., Stevenson D.E., MacDonald E.L. 2003 Biodiversity and biogeography of the islands of the Kuril Archipelago // Journal of Biogeography. Vol.30. P.1297-1310. doi: 10.1046/j.1365-2699.2003.00956.x.

Razjigaeva N.G., Ganzey L.A. 2004. [Changes of the island geosystems under the influence of catastrophic processes in the Late Pleistocene-Holocene (by the example of the Southern Kuriles] // Vestnik DVO RAN [Herald of the Far East Department of the Russian Academy of Sciences]. No.2. P.93-101 [in Russian].

Razjigaeva N.G., Ganzey L.A., Belyanina N.I., Grebennikova T.A., Ganzey K.S. 2008. Paleo-environments and landscape history of minor Kurile Islands since Late Glacial // Quaternary International. Vol.179. P.83-89.

Razjigaeva N.G., Ganzey L.A., Arslanov Kh.A., Grebennikova T.A., Belyanina N.I., Mokhova L.M. 2011. Paleoenvironments of Kurile Islands in Late PleistoceneeHolocene: climatic changes and volcanic eruption effects // Quaternary International. Vol.237. P.4-14.

Razjigaeva N.G., Ganzey L.A., Grebennikova T.A., Belyanina N.I., Mokhova L.M., Arslanov K.A., Chernov S.B. 2013. Holocene Climatic Changes and Vegetation Development in the Kurile Islands // Quaternary International. Vols290-291. P.126-138.

Ryabukhin A.S. 1991. [To the knowledge of staphylinids (Coleoptera, Staphylinidae) of the Northeastern of the USSR. I. Olophrum Erichson] // Entomologicheskie issledovanija na Severo-Vostoke SSSR. Vladivostok. Vol.2. P.11-25 [in Russian].

Ryabukhin A.S. 1999. A catalogue of rove beetles (Coleoptera: Staphylinidae exclusive of Aleocharinae) of the northeast of Asia. Sofia: Pensoft. 140 p.

Ryabukhin A.S. 2008. [Zoogeographical characteristics of the rovebeetles fauna (Insecta: Coleoptera: Staphylinidae) of Kamchatka] // Vestnik SVNC DVO RAN [Reports of North-Eastern Scientific Center of Far Eastern Branch of Russian Academy of Science]. Vol.4. P.96-100 [in Russian].

Ryabukhin A.S. 2010. [Studying rove beetles fauna (Coleoptera, Staphylinidae) in the territory of Koryakia (Kamchatka)] // Vestnik SVNC DVO RAN [Reports of North-Eastern Scientific Center of Far Eastern Branch of Russian Academy of Science]. Vol.3. P.72-77 [in Russian].

Sazhnev A.S. 2018. [Materials to the rove beetles fauna (Coleoptera: Staphylinidae) of the Commander Islands (Kamchatka Region, Russia)] // Caucasian Entomological Bulletin. Vol.14. No.1. P.19-23 [in Russian].

Schülke M., Smetana, A. 2015 Staphylinidae // I. Löbl, D. Löbl (Eds.). Catalogue of Palaearctic Coleoptera. Vol. 2. Hydrophiloidea - Staphylinoidea. Revised and Updated Edition. Leiden: Brill. P.304-1134.

Sharp D. 1889. The Staphylinidae of Japan // The Annals and Magazine of Natural History. Vol.6. No.3. P.463-476.

Shavrin A.V. 2013. A redescription of Acruliopsis tumidula (Mäklin, 1853) comb. n., with new data on A. ussuriensis Zerche, 2003 (Coleoptera: Staphylinidae: Omaliinae) // Baltic Journal of Coleopterology. Vol.13. No.2. P.125-130. 
Shavrin A.V. 2016. A review of the genus Pycnoglypta Thomson, 1858 (Staphylinidae, Omaliinae, Omaliini) with notes on related taxa // Zootaxa. Vol.4077. No.1. P.1-94.

Shavrin A.V. 2018. The lestevoides species group of the genus Geodromicus Redtenbacher, 1857 (Insecta: Coleoptera: Staphylinidae: Omaliinae) // Zootaxa. Vol.4378. No.2. P.151-190.

Shavrin A.V., Berlov E.Ya. 1999. [To the fauna of Staphylinidae (Coleoptera) of Sakhalin island] // Proceedings of the Irkutsk State Agricultural Academy. Vol.18. P.8-12 [in Russian].

Shavrin A.V., Gildenkov M.Yu. 2009. [Staphylinidae] // S.Yu. Storozhenko, Yu.N. Sundukov, A.S. Lelej, V.S. Sidorenko, M.Yu. Proshchalykin, A.N. Kupyanskaya (eds.). Insects of Lazovsky Nature Reserve. Vladivostok: Dalnauka. P.120-127 [in Russian].

Shibata Y. 1965. The staphylinid-beetles from Rishiri and Rebun isls., Hokkaido, Japan, with descriptions of three new species // Kontyû. Vol.33. No.3. P.317-323.

Shibata Y. 1976. Provisional check list of the family Staphylinidae of Japan. I (Insecta: Coleoptera) [Micropeplinae-Euasthetinae] // Annual Bulletin of the Nichidai Sanko, Tokyo. Vol.19. P.71-212.

Shibata Y., Maruyama M., Hoshina H., Kishimoto T., Naomi S.-I., Nomura S., Puthz V., Shimada T., Watanabe, Y., Yamamoto S. 2013. Catalogue of Japanese Staphylinidae (Insecta: Coleoptera) // Bulletin of the Kyushu University Museum. Vol.11. P.69-218.

Smetana A. 1981. A new species of the genus Phloeonomus Heer from the Kurile Islands (Coleoptera: Staphylinidae) // Entomologica Scandinavica. Vol.12. P.78-80.

Thayer M.K. 2016. 14.7 Staphylinidae Latreille, 1802 // R.G. Beutel, R.A.B. Leschen (eds.). Coleoptera, Beetles. Vol.1. Morphology and systematics (Archostemata, Adephaga, Myxophaga, Polyphaga partim). 2nd Edition. Handbook of zoology. Arthropoda Insecta. Berlin: Walter de Gruyter. P.394-442.

Vorobiev D.P. 1963. Vegetation of the Kurile Islands. M.-L.: USSR Academy of Science Press. 92 pp. [In Russian]

Watanabe Y. 1985. Omaliinae. // S.-I. Ueno et al. (eds.). The Coleoptera of Japan in Color 2. Hoikusha, Osaka. P.264-268.
Watanabe Y. 1988. A taxonomic study on the Japanese species of the genus Coryphium (Coleoptera, Staphylinidae) // Elytra. Vol.16. No.2. P.45-63.

Watanabe Y. 1990. A taxonomic study on the subfamily Omaliinae from Japan (Coleoptera, Staphylinidae) // Memoirs of the Tokyo University of Agriculture. Vol.31. P.59-391.

Watanabe Y., Sato M. 1981. A new Brathinus (Coleoptera, Staphylinidae) from Shikoku, Japan // Kontyû. Vol.49. No.4. P.615-619.

Watanabe Y., Shibata Y. 1965. The staphylinid-beetles from Rishiri and Rebun Isls., Hokkaido, Japan, with descriptions of three new species // Kontyû. Vol.33. No.3. P.317-323.

Weise J. 1877. Japanische Staphilinidae [sic] und Pselaphidae. Beiträge zur Käferfauna von Japan, meist auf R. Hiller's Sammulungen basirt // Deutsche Entomologische Zeitschrift. Bd.21. S.88-100.

Weise J. 1884. Ueber Eudectus rufulus n. sp. aus Japan // Deutsche Entomologische Zeitschrift. Vol.28. P.270-271.

Zanetti A. 1987. Coleoptera. Staphylinidae. Omaliinae // Fauna d'Italia. Vol.25. i-xii. P.1-472 [Calderini, Bologna]

Zanetti A. 1993. Contribution to the knowledge on eastern Palaearctic Eusphalerum Kraatz with descriptions of new species (Coleoptera, Staphylinidae: Omaliinae) // Annales Historico-naturales Musei Nationalis Hungarici. Vol.85. P.47-63.

Zerche L. 1990. Monographie der paläarktischen Coryphiini (Coleoptera, Staphylinidae, Omaliinae). Akademie der Landwirtschaftswissenschaften der Deutschen Demokratischen Republik. S.1-413.

Zerche L. 1993. Monographie der paläarktischen Coryphiini (Coleoptera, Staphylinidae, Omaliinae). Supplementum $1 / / \mathrm{Be}-$ iträge zur Entomologie. Bd.43. H.2. S.319-374.

Zerche L. 2003. Arten der Hygrogeus-Gruppe aus dem Fernen Osten Russlands, aus Japan und China (Coleoptera, Staphylinidae, Omaliinae, Anthophagini) // Beiträge zur Entomologie. Bd.53. H.2. S.277-295. 